\title{
Attention control: The missing link between sensory discrimination and intelligence
}

\author{
Jason S. Tsukahara ${ }^{1}$ (D) Tyler L. Harrison ${ }^{2} \cdot$ Christopher Draheim $^{1} \cdot$ Jessie D. Martin $^{1} \cdot$ Randall W. Engle $^{1}$ \\ Published online: 8 June 2020 \\ (C) The Psychonomic Society, Inc. 2020
}

\begin{abstract}
Intelligence is correlated with the ability to make fine sensory discriminations. Although this relationship has been known since the beginning of intelligence testing, the mechanisms underlying this relationship are still unknown. In two large-scale structural equation-modelling studies, we investigated whether individual differences in attention control abilities can explain the relationship between sensory discrimination and intelligence. Across these two studies, we replicated the finding that attention control fully mediated the relationships of intelligence/working-memory capacity to sensory discrimination. Our findings show that attention control plays a prominent role in relating sensory discrimination to higher-order cognitive abilities.
\end{abstract}

Keywords Attention control · Sensory discrimination · Intelligence · Working memory

\section{Introduction}

The act of making a sensory discrimination is quite simple. You hear two tones and must decide which tone is of a higher pitch, or you see two vertical lines and must decide which line is longer. Sensory discrimination can occur in a variety of modalities such as differences in weight between objects, light brightness, or tone loudness. On the surface, this may seem like a task that simply requires basic sensory processing. Auditory processing for pitch discrimination, visual processing for line discrimination, tactile processing for weight, and so on. However, over 130 years ago Francis Galton (1883) hypothesized that "the more perceptive the senses are of difference, the larger is the field upon which our judgment and intellect can act." (p. 19). Not long thereafter, Charles Spearman (1904) showed this to be true; sensorydiscrimination ability correlated with intelligence. What was so striking about Spearman's discovery was not just that specific sensory modalities (e.g., auditory or visual) correlated with intelligence, but that what was common across the modalities, a domain-general sensory-discrimination ability,

Jason S. Tsukahara

jason.tsukahara@gatech.edu

1 School of Psychology, Georgia Institute of Technology, Atlanta, GA 30332, USA

2 Department of Psychology, University of North Georgia, Dahlonega, GA, USA correlated nearly perfectly $(r=1)$ with general intelligence. Despite this strong correspondence, there has been little to no theoretical development as to why sensory discrimination correlates so strongly with intelligence. Our position is that attention control, one's ability to guide the focus of attention in a goal-directed manner and to reduce distraction, is an important determinant of individual differences on intelligence and likely plays an important role in sensory discrimination. Therefore, in two studies we investigated whether individual differences in attention control can account for the relationship between sensory discrimination and intelligence.

The discovery of the sensory discrimination - intelligence relationship coincided with a pivotal moment in the history of intelligence testing: the development of more rigorous quantitative methods to measure the degree of correlation between measures. While the statistical methods demonstrated by Spearman (1904) became the precursor to modern statistical methods, psychologists discontinued research on the relation between sensory discrimination and intelligence (Deary, 1994b). It would not be until after the field shifted towards the information-processing paradigm that researchers started showing interest in the sensory discrimination and intelligence relationship again. Starting around the 1980s, researchers once again showed that measures of sensory discrimination correlated with measures of intelligence (Acton \& Schroeder, 2001; Helmbold et al., 2006; Lynn et al., 1989; Raz et al., 1983, 1987, 1990; Watson, 1991). These studies found individual task correlations in the range of $r=.2-.5$. Not until 100 years after Spearman (1904) was the latent correlation (as 
compared to single-task correlations) between sensorydiscrimination ability and intelligence tested again. When tested at the latent construct level, studies have shown an even stronger correlation between sensory discrimination and general intelligence, with a range of $\mathrm{r}=.61-.95$ (Deary et al., 2004; Meyer et al., 2010; Troche et al., 2014).

Spearman considered general intelligence (g) as an overarching factor that represented common variance amongst disparate tests of mental abilities. Spearman (1904) theorized that the reason disparate tests of mental abilities were correlated with one another was due to some central Function. Based on his findings of a near-perfect correlation between sensory discrimination and general intelligence, Spearman (1904) concluded that,

Finally, there is the exceedingly significant fact that this central Function, whatever it may be, is hardly anywhere more prominent than in the simple act of discriminating two nearly identical tones... Discrimination has unrivalled advantages for investigating and diagnosing the central Function (Spearman 1904, p. 274).

Therefore, despite the lack of research on the sensory discrimination - intelligence relationship Spearman considered sensory discrimination as vital to understanding the nature of general intelligence and this central Function.

Various hierarchical models of general intelligence have been proposed since Spearman (1904) that either dismiss the existence of $g$ in place of separate mental abilities or subsume second-order mental abilities under an over-arching $g$ factor (H. Kane \& Brand, 2003). The Hebb-Cattell theory of intelligence, first proposed by Hebb (1942), ${ }^{1}$ distinguishes between fluid intelligence and crystallized intelligence. Where fluid intelligence is the ability to reason, learn, and adaptively form new patterns of behavior in novel situations. Hebb conceptualized this as a sort of intellectual power or intellectual efficiency that leads to the acquisition of knowledge, and new perceptual/behavioral patterns. Crystallized intelligence is the functioning of already acquired knowledge and developed perceptual/behavioral patterns.

In any test performance there are two factors involved, one factor being present intellectual power, of the kind essential to normal intellectual development; the other being the lasting changes of perceptual organization and

\footnotetext{
${ }^{1}$ Traditionally, the fluid/crystallized intelligence model has been credited to Raymond B. Cattell. For an interesting investigation into the origin of the fluid/crystallized intelligence distinction, see Brown (2016). The author concludes that Cattell adopted and extended Donald O. Hebb's theory of two intelligences and termed them names fluid and crystallized intelligence. As such, the author suggests that it should be called the Hebb-Cattell Theory.
}

behavior induced by the first factor during the period of development (Hebb 1942 p. 287).

Historically, attention-related processes have been underemphasized in models of fluid intelligence (H. Kane \& Brand, 2003) and in psychophysical approaches to sensation and perception (the role of attention is neglected in the formative book on psychophysics by Stevens, 1975). However, more recent models of intelligence have included attention control as a primary factor contributing to individual differences in fluid intelligence (Conway et al., 1999, 2003; Heitz et al., 2005; Kovacs \& Conway, 2016; Kyllonen \& Christal, 1990; Shipstead et al., 2016). And more recent neural models of perception have proposed attention as an important mechanism at early and late stages of perception (Deco \& Rolls, 2005; Desimone \& Duncan, 1995; Kok, 1997; Luck et al., 2000; O'Craven et al., 1997; Usher \& Niebur, 1996). Therefore, attention processes may play an important role in relating lower-level sensory abilities to higher-order cognitive abilities.

Attention control is the general ability to engage executive control functions that guide the focus of attention in a goaldirected manner (Barrett et al., 2004; M. J. Kane et al., 2001; Posner \& DiGirolamo, 1998; Rueda, 2018). The focus of attention can be influenced by many processes; a traditional classification scheme is to distinguish between automatic (bottom-up, habitual, reflexive, or stimulus-driven) and controlled (top-down, or goal-directed) processes (Egeth \& Yantis, 1997). If automatic processes direct attention in a manner consistent with current goals or a desirable level of performance, then there is less need to engage in attention control (Norman \& Shallice, 1986). However, if there is conflict between automatic processes and current goals or performance level, then there arises the need to engage attention control to guide thoughts and behavior in a more purposeful and goaldirected manner (Norman \& Shallice, 1986). Individual differences in attention control manifest when automatic processes are in conflict with goal-oriented processes (M. J. Kane \& Engle, 2003). Commonly used measures of attention control often include the need to resolve conflict, reduce distractor interference, or inhibit prepotent responding. As such, the construct of attention control has often been defined by aspects of selection or inhibition (Friedman \& Miyake, 2004).

However, an often-overlooked aspect of attention control is that of the intensity of attention. The intensity of attention can generally be understood as the degree or amount of attention allocated to a task, stimulus, visual location, etc. The distinction between selective and intensive aspects of attention has been discussed in psychology since William James (1890); "Everyone knows what attention is. It is taking possession of the mind, in clear and vivid form, of one out of what seems several simultaneously possible objects or trains of thought." "In clear and vivid form" refers to the intensive aspect of 
attention; the more intensively attention is applied, the more clear and vivid will be the perception of the selected object or train of thought. Kahneman (1973) states "There is more to attention than mere selection... the term 'attention' also refers to an aspect of amount and intensity." The selective and intensity aspects of attention should not necessary be considered as independent of one another. For instance, selectively attending to certain stimuli over others will afford a greater intensity of attention to selected stimuli and less intensity of attention to unselected stimuli; although this is likely just a reflection of the simple fact that attention is always selective. However, within the selection or focus of attention the intensity of attention can vary; one can attend to the items in the focus of attention with a high or low degree of vividness. In fact, much of the early cognitive neuroscience work focused on understanding the effects of top-down attention on early perceptual processing. This research lead to the understanding that attention leads to the enhancement and sensitivity of neuronal response in early perceptual brain regions to bias neural competition towards goal-relevant behavior (Desimone \& Duncan, 1995; Reynolds \& Pasternak, 2000). Additionally, recent work by Unsworth and colleagues has suggested that the voluntary control of the intensity of attention is important for understanding the relationship between working-memory capacity and attention control (A. L. Miller et al., 2019; Unsworth \& Robison, 2017, 2020).

This broader view of attention control as more than just selection or inhibition is a reflection of our executive attention view of cognition in which executive attention organizes processing requirements around task-objectives or current goals (Shipstead et al., 2016). Processing requirements will depend on the nature of the task; some tasks may place a greater demand on selection, on intensity, and on other attentionrelated processes. Therefore, attention control is not defined by any one specific mechanism or process but rather acts to organize and modulate processes around a particular goal. Originally, we presented this executive attention view as a way to explain the relationship between fluid intelligence and working-memory capacity (Shipstead et al., 2016). Working-memory capacity, a construct closely related to attention control, is known to correlate at the latent construct level very highly with fluid intelligence, typically in the range of .6-.8 (M. J. Kane et al., 2005). Individual differences in working-memory capacity reflect a domain-general ability to maintain and manipulate information in an accessible state in the face of interference and distraction. It is distinct from short-term memory in that there is the added demand to reduce interference from irrelevant information or distracting stimuli. As such, working-memory capacity has been shown to predict fluid intelligence over and above short-term memory (Engle, 2002). The three constructs; fluid intelligence, attention control, and working-memory capacity, are all highly correlated with one another but also show unique variance (Heitz et al.,
2006). Our executive attention view states that individual differences in attention control can account for the shared relation between fluid intelligence and working-memory capacity (Engle, 2018; Shipstead et al., 2016). That is, the reason fluid intelligence and working-memory capacity are so highly related is that the tasks used to measure these constructs place a strong demand on functions of attention control.

The extent to which attention control plays a role in sensory discrimination has been under-emphasized in previous research. However, Troche et al. (2014) have shown that working-memory capacity can explain the relationship between sensory discrimination and fluid intelligence, suggesting that mechanisms of attention control likely play an important role. It is also known that top-down processes can enhance neural activation in sensory areas and bias processing of target stimuli (Aston-Jones \& Cohen, 2005; Reynolds \& Pasternak, 2000; Schoenfeld et al., 2007). One possibility is that to perform a sensory discrimination requires processes in working memory that rely heavily on attention control. For instance, the comparison of two stimuli on a single dimension (e.g., two tones of different pitch) requires maintaining a mental representation of the two stimuli in working memory. The strength of the mental representation will depend on several processes, including initial encoding of the stimuli and maintenance in working memory as attention shifts from one stimulus to the other. We would argue that both these processes rely heavily on attention control. Admittedly, if previous research had not shown that sensory discrimination correlates strongly with working-memory capacity and intelligence there would be little a priori reason to expect attention control to play such a strong role in sensory discrimination. However, the correlation of sensory discrimination with these broad domain-general cognitive abilities suggests that the act of sensory discrimination requires more than bottom-up perceptual attention processes and likely requires some degree of controlled attention.

This all suggests that a unitary domain-general faculty of attention control should contribute to individual differences in sensory-discrimination ability and its relation to fluid intelligence and working-memory capacity. Therefore, we would argue that the processes underlying sensory discrimination rely heavily on attention control and hypothesize that individual differences in attention control can explain the relationship between sensory discrimination and fluid intelligence/ working-memory capacity.

In two studies, we used confirmatory factor analysis and structural equation modelling to test our hypothesis. The latent constructs in the two studies were sensory discrimination, attention control, working-memory capacity, and fluid intelligence. Each latent construct was measured using multiple tasks. The procedure for the sensory discrimination tasks used in the current studies was similar to those used by other researchers (Troche et al., 2014) and employed an adaptive 
procedure to estimate a discrimination threshold value (Kaernbach, 1991). Study 1 included both visual and auditory discrimination tasks, while Study 2 included only auditory discrimination tasks but included improved measures of attention control.

\section{Study 1}

The purpose of Study 1 was to test our hypothesis that attention control can explain the relationship between sensory discrimination and working-memory capacity/fluid intelligence. We measured sensory discrimination using two visual (line length and circle size) and two auditory (loudness and pitch) discrimination tasks. We used structural equation modelling to test whether attention control mediates the relationship between sensory discrimination and working-memory capacity/fluid intelligence.

\section{Method}

\section{Subjects}

The study consisted of four 2-hour sessions. Subjects were college students and non-college adults of the Atlanta community. All subjects were required to be native English speakers (i.e., learned English before the age of 5 years), 18-35 years of age, and had not participated in a study with our lab before. No screening on vision or hearing was performed. Subjects were compensated with a $\$ 30$ check at the end of each session and received an additional $\$ 10$ check as a compensation bonus at the end of the fourth session. There was also an option for Georgia Tech students to receive $2 \mathrm{~h}$ of course credits instead of monetary compensation on each session. If they chose course credit for some or all of the sessions, they still received the $\$ 10$ check as a compensation bonus for completing all four sessions. This study was approved by the Georgia Institute of Technology's Institutional Review Board.

A total of 355 subjects completed all four sessions, and 24 subjects were removed due to having excessive missing data, for a final sample size of 331. Monte-Carlo simulations suggest that for stable estimates of correlations sample size should approach 250 (Schönbrodt \& Perugini, 2013). Excessive missing data was defined as having missing data on more than half of the task indicators for any latent construct. A subject may have missing data on a task for a variety of reasons including lost data file, task crashed before they could finish, or they did not meet a certain criterion of performance. The criteria of performance are defined on a task-by-task basis and are described in more detail in the Data processing and analysis section. Table 1 displays the demographics for the 331 subjects.
Table 1 Subject demographics for Study $1(\mathrm{~N}=331)$

\begin{tabular}{ll}
\hline Demographic & Statistic \\
\hline Age (years) & Mean: 24.5 \\
& SD: 4.7 \\
Gender & Male: $50 \%$ \\
& Female: $50 \%$ \\
College student $^{1}$ & Yes: $49 \%$ \\
& No: $51 \%$ \\
Ethnicity $^{2}$ & White: $62 \%$ \\
& Black or African American: 19\% \\
& Asian or Pacific Islander: $10 \%$ \\
& Other: $9 \%$ \\
\hline
\end{tabular}

${ }^{1}$ College students included Georgia Tech and other colleges in Atlanta

2 "Other" includes Native American, Hispanic or Latino, and other

\section{Tasks and procedures}

Subjects performed a battery of cognitive tasks in a group running room. The group running room had five subject stations, and one research assistant monitored subjects. The tasks were performed on a Windows 7 computer with an LEDbacklit LCD monitor, and subjects wore headphones during all tasks. The tasks were programmed in E-Prime 2.0.10 software (Psychology Software Tools, Inc. [E-Prime 2.0], 2016).

Working-memory capacity We measured working-memory capacity using three complex span tasks; the advanced operation span, advanced symmetry span, and advanced rotation span. The complex span tasks consist of alternating memory storage and processing sub-tasks (Unsworth et al., 2005). The advanced versions of the tasks included larger set-sizes of memory items (Draheim et al., 2018). In all complex span tasks, the total number of items recalled in their correct serial position (partial score) was used to calculate scores on the task (Conway et al., 2005).

Advanced operation span. This task required subjects to remember a series of letters presented in alternation with simple math equations that they were required to solve. On each trial, subjects first solved a simple math equation followed by the presentation of a single letter. This alternation repeated until a variable set-size of letters-to-beremembered had been presented. Then, on the recall screen subjects had to recall the letters in the correct order by clicking the mouse on the appropriate letters from a matrix of letters displayed on the screen. There was a total of 14 trials (two blocks of seven trials), set-sizes ranged from $3-8,{ }^{2}$ and each set-size occurred twice (once in each

\footnotetext{
${ }^{2}$ Due to an error in programming of the advanced operation span task, trials in which the set-size was supposed to be nine actually only displayed a set-size of eight. This resulted in the set-size of eight occurring on a total of four trials, compared to two trials for all other set-sizes.
} 
block). Scores on the advanced operation span task were calculated using the partial-scoring method, the number of letters recalled in their correct order across all trials.

Advanced symmetry span. This task required subjects to remember a series of spatial locations in a $4 \times 4$ matrix presented in alternation with a pattern of squares for which they had to decide whether the pattern was symmetrical on the vertical midline. On each trial, subjects were first presented with a $16 \times 16$ matrix of black and white squares and were required to decide whether the pattern was symmetric on the vertical midline. Followed by the symmetry judgment, a $4 \times 4$ matrix of squares with one square highlighted in red were displayed. The location of the red square was the to-be-remembered spatial location. This alternation continued until a variable setsize of spatial locations had been presented. Then, on the recall screen the same $4 \times 4$ matrix of squares was presented but with no squares highlighted in red. Subjects had to recall the spatial locations in the correct order by clicking the mouse on the appropriate squares in the matrix. There was a total of 12 trials (two blocks of six trials), set-sizes ranged from two to seven, and each setsize occurred twice (once in each block). Scores on the advanced symmetry span task were calculated using the partial-scoring method, the number of spatial locations recalled in their correct order across all trials.

Advanced rotation span. This task required subjects to remember a series of directional arrows of varying size in alternation with a mental rotation task in which they had to mentally rotate and decide if a letter was mirror reversed or not. On each trial, subjects first solved a mental rotation problem followed by the presentation of a single arrow with a specific direction (eight possible directions; the four cardinal and four ordinal directions) and specific size (small or large). Both the direction and size of the arrow were the to-be-remembered features. This alternation continued until a variable set-size of arrows had been presented. Then, on the recall screen all possible arrow directions and sizes were presented. Subjects had to recall the direction and size of the arrows in the correct order by clicking the mouse on the appropriate arrow. There was a total of 12 trials (two blocks of six trials), set-sizes ranged from two to seven, and each set-size occurred twice (once in each block). Scores on the advanced rotation span task were calculated using the partial-scoring method, the number of arrows recalled in their correct order across all trials.

\section{Fluid intelligence}

Raven's advanced progressive matrices. For each problem in this task, subjects were presented with a $3 \times 3$ matrix of eight abstract figures with the bottom-right element missing (Raven et al., 1998). This matrix of figures followed a particular logical pattern. Subjects had to identify the logical pattern and select one of eight answer choices that fitted the logical pattern as the rest of the matrix. Subjects were given $10 \mathrm{~min}$ to solve the 18 odd problems. Their score on this task was the total number of problems solved correctly.

Letter sets. For each problem in this task, subjects were presented with five sets of four-letter sequences (Ekstrom et al., 1976). Four of these sets followed a particular rule (e.g., four sets are in alphabetical order, four sets have the letter " $X$ " in the third location). Subjects had to identify what the rule was and select the set of letters that did not follow that rule. Subjects were given 10 min to solve 30 problems. Their score on this task was the total number of problems solved correctly.

Number series. For each problem in this task, subjects were presented with a series of numbers (Thurstone, 1938). These numbers progressed in a particular logical fashion. Subjects had to identify the rule and select the next number that should appear in the series of numbers to be consistent with the logic out of five answer choices. Subjects were given $5 \mathrm{~min}$ to complete 15 problems. Their score on this task was the total number of problems solved correctly.

\section{Attention control tasks}

Antisaccade. In this task, subjects had to identify a "Q" or "O" that appeared very briefly on the opposite side of the screen as a distractor stimulus (Hallett, 1978; Hutchison, 2007). Subjects were first presented with a fixation cross at the center of the screen. After a 1,000$\mathrm{ms}$ or 2,000-ms interval an asterisk (*) flashed at $12.3^{\circ}$ visual angle to the left or right of the central fixation for $100 \mathrm{~ms}$. After presentation of the asterisk, either "Q" or "O" was presented on the opposite side at $12.3^{\circ}$ visual angle of the central fixation for $100 \mathrm{~ms}$ quickly followed by a visual mask (\#\#). Subjects had to indicate whether the letter was a "Q" or an "O." Subjects completed 16 slow practice trials (target duration was set to $750 \mathrm{~ms}$ ). There were 48 trials and the task score was calculated as the proportion of correct trials.

Arrow flanker. In this task subjects had to identify the direction (left or right) of a central arrow that was flanked by arrows that were either in the same or the opposite direction (Shipstead et al., 2014). On each trial, subjects were first presented with a fixation point in the center of the screen that lasted $900 \mathrm{~ms}$. After the fixation, a horizontal array of five items appeared in the center of the screen. The central item was always an arrow facing to either the left or the right. Subjects had to indicate the direction of the center arrow as quickly and accurately 
as possible by pressing the "z" (left) or " " (right) key. The items of the array beside the center arrow could be facing the same direction as the center arrow (i.e., congruent trials), facing the opposite direction as the center arrow (i.e., incongruent trials), or be horizontal lines (i.e., neutral trials). There was a total of 216 trials with 72 trials of each category (e.g., incongruent). The score on the Flanker task was calculated by subtracting the mean reaction time on congruent trials from the mean reaction time on incongruent trials. Only accurate trials were used to in calculating mean reaction times.

Color Stroop. For this task subjects were presented with a word (either "RED", "GREEN", or "BLUE") and had to indicate the hue in which the word was printed (red, green, or blue). On each trial, there was first a central fixation $(400-700 \mathrm{~ms})$ followed by a centrally presented word. The subject made a response on a keyboard using three keys labeled with the colors (green, blue, and red). Subjects had to complete 486 trials. For $66 \%$ of the trials, the hue and the word were congruent. For the other 33\% of the trials, the hue and the word were incongruent. The score on the Stroop task was calculated by subtracting the mean reaction time on congruent trials from the mean reaction time on incongruent trials. Only accurate trials were used to in calculating mean reaction times.

Sensory discrimination The sensory discrimination tasks required subjects to discriminate the difference between two sensory stimuli. We used a weighted up-down adaptive procedure with 64 trials to estimate a difference threshold that converged on a certain level of performance (Kaernbach, 1991). If subjects made a correct discrimination, the difference between the two stimuli decreased (more difficult discrimination) on the next trial. If the subject made an error, the difference between the two stimuli increased (easier discrimination) on the next trial. The ratio to decrease/increase the difference was 1:3. This weighted up-down method with a step ratio of 1:3 converges around a threshold value of $75 \%$ (Kaernbach, 1991). To estimate a threshold value we employed a method of averaging the difference value for the last four reversals (Hairston \& Maldjian, 2009; although they used the last five reversals). A reversal is when the accuracy on the current trial is different from the accuracy on the previous trial. See Fig. 1 for an example of the adaptive procedure and scoring method for two real subjects on the pitch discrimination task. The critical dependent measure on all sensory discrimination tasks is the average difference value of the last four reversals.

There are several different ways researchers have calculated the threshold value from psychophysical adaptive tasks. While we report the results based on the average of the last four reversals, we conducted the analyses using an average across all trials (Kaernbach, 1991), an average of the last 10-20 trials (Rammsayer, 1992), and the difference arrived at on the last trial. Regardless of which dependent measure we used for the task, overall the results were the same. Obviously, there were differences in task loadings, bivariate and latent correlations, and regression paths in the structural models, but these differences were minor, and the interpretation of the models did not change.

Line discrimination. For this task subjects were presented with two lines on the computer monitor and had to determine which line was longer (see Fig. 2 for an example trial). The lines were displayed in the upper left and in the lower right quadrants of the screen. The lines were $8.5 \mathrm{~cm}$ apart from each other horizontally. The exact vertical locations of these lines were randomly jittered from 0 to $2 \mathrm{~cm}$. Subjects had unlimited time to decide which line was longer and the lines were visible on the screen while subjects made their decision. For half of the trials the line on the left was longer and for the other half the line on the right was longer.

The first trial started at a line length difference of 25 pixels. For all trials, the line length difference decreased (after correct trials) by 5 pixels and increased (after incorrect trials) 15 pixels. The minimum line length difference was 1 pixel and the maximum line length difference was 55 pixels. There was a total of 64 trials.

Circle discrimination. This task was structurally similar to the line discrimination task, but subjects were presented with two circles and had to determine which circle was larger (see Fig. 3 for an example trial). The circles were displayed in the upper left and lower right quadrants of the screen and were approximately $8.5 \mathrm{~cm}$ apart from each other horizontally. The exact vertical locations of these circles were randomly jittered from 0 to $2 \mathrm{~cm}$. Subjects had unlimited time to decide which circle was larger and circles were visible on the screen while subjects made their decision. For half of the trials the circle on the left was larger and for the other half the circle on the right was larger.

The first trial started at a circle diameter difference of 10 pixels. For the first six trials the circle diameter difference decreased (after correct trials) by 2 pixels and increased (after incorrect trials) by 6 pixels. After the first six trials the circle diameter difference decreased by 1 pixel and increased by 3 pixels. The minimum circle diameter difference was 1 pixel and the maximum circle diameter difference was 55 pixels. There was a total of 64 trials. 


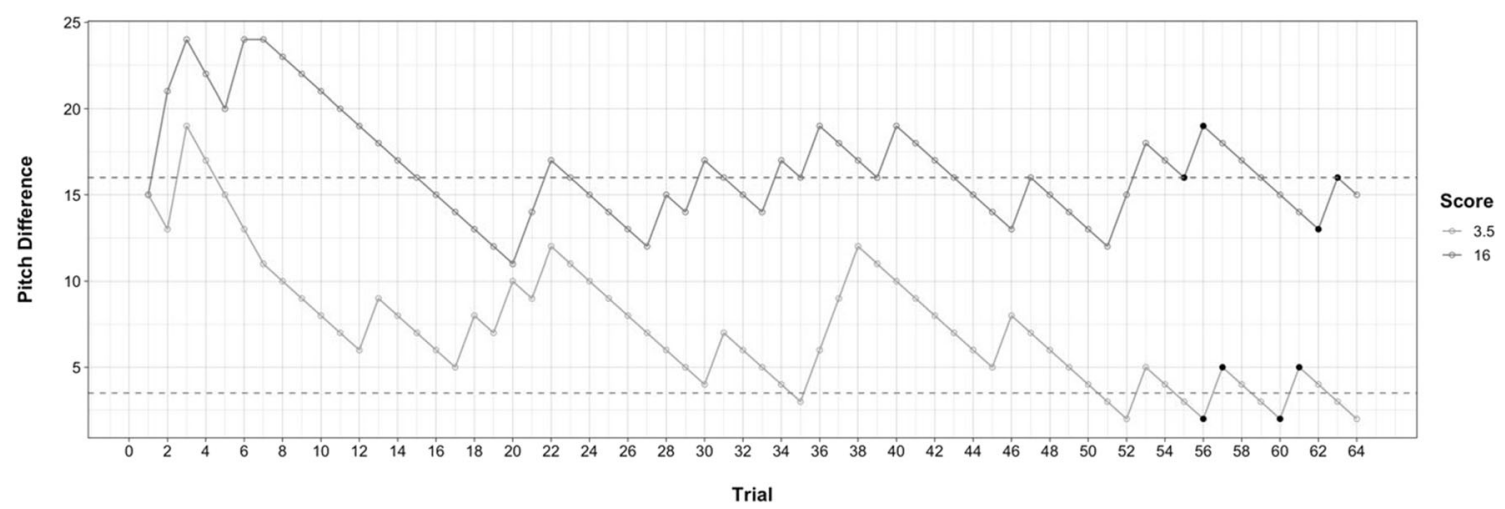

Fig. 1 An example of the adaptive procedure and scoring method for two real subjects on the pitch discrimination task. Each point represents the pitch difference between the two tones (y-axis) on each trial (x-axis). The difference gets smaller (goes down) on the next trial if the subject makes a correct response and gets larger (goes up) if they make an incorrect response at a 3:1 up:down ratio. Reversals are the peaks (a switch from

Pitch discrimination. For this task subjects were presented with two auditory tones and indicated which tone had a higher pitch. Each tone was presented for $500 \mathrm{~ms}$ with a 500-ms interstimulus interval. After the tones had played, the subject was given unlimited time to decide which tone had a higher pitch. All tones were pure and were between $776 \mathrm{~Hz}$ and $864 \mathrm{~Hz}$. All tones had a 5-ms fade-in at the beginning of the tone and a 5$\mathrm{ms}$ fade-out at the end of the tone. For half of the trials the first tone had a higher pitch and for the other half the second tone had a higher pitch.

The first trial started at a pitch difference of $15 \mathrm{~Hz}$. For the first six trials the pitch difference decreased (after correct trials) by $2 \mathrm{~Hz}$ and increased (after incorrect trials) by $6 \mathrm{~Hz}$. After the first six trials the pitch difference decreased by $1 \mathrm{~Hz}$ and increased by $3 \mathrm{~Hz}$. The minimum pitch difference was $1 \mathrm{~Hz}$ and the maximum pitch difference was $24 \mathrm{~Hz}$. There was a total of 64 trials.

Loudness discrimination. For this task, subjects were presented with two auditory sine wave tones and had to indicate which tone was louder. Each tone was presented for $500 \mathrm{~ms}$ with a 500-ms interstimulus interval. After the

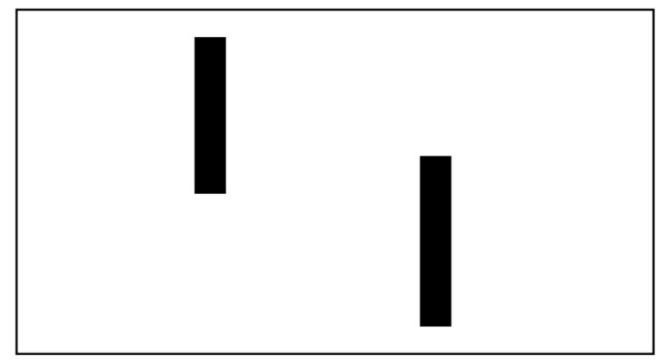

Fig. 2 An example of a line discrimination task. The subject has to indicate which line is longer. In this example, the line on the right is longer increasing to decreasing) and valleys (a switch from decreasing to increasing). The trial series for two subjects are displayed with their estimated threshold score on the pitch discrimination task given in the legend and represented by the horizontal dashed lines. The four black points are that subject's last four reversals. Their score is calculated by averaging the pitch difference of those last four reversals

tones had played, the subject was given unlimited time to decide which tone was louder. All tones were pure and were between $55 \mathrm{~dB}$ and $70 \mathrm{~dB}$. All tones had a 5-ms fade-in at the beginning of the tone and a 5-ms fade-out at the end of the tone. For half of the trials the first tone was louder and for the other half the second tone was louder.

The first trial started at a loudness difference of $10 \mathrm{~dB}$. For the first six trials the loudness difference decreased (after correct trials) by $2 \mathrm{~dB}$ and increased (after incorrect trials) by 6 $\mathrm{dB}$. After the first six trials the loudness difference decreased by $1 \mathrm{~dB}$ and increased by $3 \mathrm{~dB}$. The minimum loudness difference was $1 \mathrm{~dB}$ and the maximum loudness difference was $13 \mathrm{~dB}$. There was a total of 64 trials.

\section{Data processing and analysis}

Scoring criteria For tasks that rely on reaction time (flanker and Stroop), we removed trials that had too short reaction times to accurately reflect task processing. If reaction times were shorter than $200 \mathrm{~ms}$, then the trial was removed. Mean reaction times were then calculated based on the remaining accurate trials only. If the subject's mean accuracy on

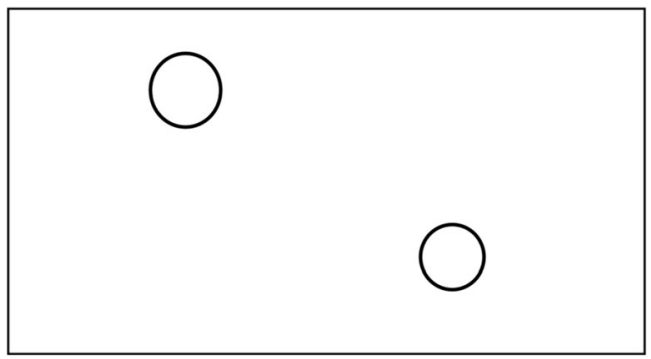

Fig. 3 This figure shows an example of the circle discrimination task. The subjects would have to indicate whether the circle on the left or right was larger. In this example, the circle on the left is larger 
congruent or on incongruent trials was 3.5 standard deviations (SDs) below the mean, their score on the task was removed.

For the complex span tasks, if a subject performed at less than $50 \%$ accuracy on the processing portion of the task, then their score for that task was set to missing (a stricter criteria of $80 \%$ accuracy resulted in removing too many subjects). For the sensory discrimination tasks, if the subject's mean accuracy was 3.5 SDs below the mean, then their score on the task was removed. For every task, we then removed univariate outliers by removing task scores that were 3.5 SDs above or below the mean for that task.

When measuring psychological variables at the latent construct level it is important to use multiple indicators of that construct. For this reason, if a subject had missing or removed data on more than half of the task indicators for any one construct they were completely removed from any further analyses. After using these scoring criteria, a total of 24 subjects were removed from further analyses resulting in a final sample size of 331.

Data analysis Reliability estimates are provided for each task. Reliability for all the tasks except the sensory discrimination tasks were calculated using split-half corrected for with Spearman-Brown prophecy formula. For the Flanker and Stroop tasks, we split congruent trial types into even and odd trials and then did the same for incongruent trial types. We then computed the mean reaction time on congruent and incongruent trial types separately for even and odd trials, followed by calculating the difference between congruent and incongruent trials separately for even and odd trials. This provides an interference effect for even trials and an interference effect for odd trials. The split-half reliability for Flanker and Stroop tasks was, therefore, calculated as the correlation, corrected with Spearman-Brown prophecy formula, between the even and odd interference effects. Because the sensory discrimination tasks were adaptive, this means the trials administered across the task are dependent on each other. That is, performance on a current trial determines the difficulty, and thereby performance, on the next trial. Also, the adaptive task scores were not an aggregate performance across all trials. They were, however, an aggregate of the discrimination difference (e.g., pitch difference between the two tones) of the last four reversals. Therefore, to calculate the reliability of the sensory discrimination tasks we calculated Cronbach's alpha of the last four reversals (we did not use split-half reliability because there were only four trials, which therefore may give rise to more noisy estimates of reliability).

We used structural equation modelling to test our hypotheses concerning the relationship amongst the latent constructs; sensory-discrimination ability, fluid intelligence, workingmemory capacity, and attention control. First, we used confirmatory factor analysis to test whether the tasks are good measures of each construct and assess the correlations amongst the four constructs. We then tested our hypothesis that attention control mediates the relationship between fluid intelligence/ working-memory capacity and sensory-discrimination ability using structural equation models. All analyses were conducted in R statistical software ( $\mathrm{R}$ Core Team, 2018). The $\mathrm{R}$ package lavaan (Rosseel, 2012) was used for all confirmatory factor analyses and structural equation models, treatment of missing values was set to full-information maximum likelihood.

For all structural equation models, solid paths represent significant paths and dotted lines represent non-significant paths. The chi-square values, degrees of freedom, and chisquare significance are reported. The chi-square assesses overall fit and discrepancy between the sample and generalized population-wide fitted covariance matrices. Although a nonsignificant chi-square value is preferred, indicating it is not different from a general population model, it is very sensitive to sample size. As such, the chi-square value alone is not sufficient to accept or reject a model. Models must be considered in holistic terms based on multiple fit indices. The following fit indices are reported as well: The confirmatory fit indices (CFIs) and the Root Mean Square Error of Approximation (RMSEA). The CFI compares model fit to a null model. Models with a CFI $>.90$ are considered an acceptable fit, and with a CFI of .95 or higher considered to be a good fit (Hu \& Bentler, 1999). The RMSEA is a parsimony adjusted fit index. Models with an RMSEA $<.08$ are considered to be an acceptable fit, with an RMSEA of .05 or lower considered to be a good fit (Browne \& Cudeck, 1993; Kenny, 2015). Any models that are not considered as an acceptable or good fit are thereby considered as a poor model fit.

\section{Results}

Data, analysis scripts, and results output are available via the Open Science Framework: https://osf.io/hsqru/. The descriptive statistics for the individual tasks are presented in Table 2. The task-level correlation matrix is presented in Table 3. Even at the task-level the sensory discrimination tasks showed anywhere from small to high correlations with the other cognitive tasks. The sensory discrimination tasks correlated with each other moderately well, with loudness discrimination having the highest correlations with the other discrimination tasks.

\section{Measurement model}

First, we conducted an exploratory factor analysis to investigate the latent structure of the tasks in a data-driven manner. We used principal axis factoring with a varimax rotation. Visual inspection of the scree plot indicated at least two factors. Three factors had eigenvalues greater than 1 . Therefore, we specified a model with three factors and a model with two factors. The three-factor model is presented in Table 4. The 
Table 2 Descriptive statistics for each task in Study $1(\mathrm{~N}=331)$

\begin{tabular}{|c|c|c|c|c|c|}
\hline & Mean (SD) & Min-max & Skewness & Kurtosis & $\%$ Missing \\
\hline \multicolumn{6}{|l|}{$\mathbf{G f}(A C C)$} \\
\hline RAPM & $8.7(3.7)$ & $1-17$ & 0.17 & -0.88 & 1.8 \\
\hline LetterSets & $14.4(4.8)$ & $2-26$ & -0.04 & -0.39 & 2.1 \\
\hline NumberSeries & $8.0(3.2)$ & $1-15$ & 0.32 & -0.76 & 3.6 \\
\hline \multicolumn{6}{|l|}{$\mathbf{W M C}(A C C)$} \\
\hline SymSpan & $23.3(10.2)$ & $0-54$ & 0.23 & -0.28 & 3.6 \\
\hline OSpan & $49.0(18.3)$ & $1-82$ & -0.34 & -0.64 & 2.4 \\
\hline RotSpan & $21.1(9.8)$ & $0-47$ & 0.19 & -0.32 & 4.5 \\
\hline \multicolumn{6}{|l|}{ Attention } \\
\hline Antisaccade (ACC) & $0.79(0.17)$ & $0.33-1.0$ & -0.72 & -0.58 & 0.3 \\
\hline Flanker $(R T)$ & $95.7(42.5)$ & $14.7-252.8$ & 0.83 & 0.77 & 4.2 \\
\hline Stroop $(R T)$ & $153.0(96.4)$ & $-49.7-500.6$ & 0.65 & 0.70 & 3.3 \\
\hline \multicolumn{6}{|l|}{ Discrimination } \\
\hline Pitch $(H z)$ & $16.2(6.6)$ & $2.5-23.5$ & -0.49 & -1.15 & 2.4 \\
\hline Loudness $(d B)$ & $6.1(3.0)$ & $2.5-12.5$ & 0.65 & -0.86 & 3.6 \\
\hline Line $(p x)$ & $13.9(5.5)$ & $8.5-35.0$ & 1.48 & 2.35 & 1.5 \\
\hline Circle $(p x)$ & $3.2(0.9)$ & $2.5-7.0$ & 1.87 & 4.14 & 2.7 \\
\hline
\end{tabular}

$G f$ fluid intelligence, $W M C$ working-memory capacity, $A C C$ accuracy, $R T$ reaction-time interference effect, $H z$ hertz, $d B$ decibels, $p x$ pixels

Gf and WMC tasks were calculated as total correct. Antisaccade was calculated as proportion correct

fluid intelligence tasks loaded heavily on the first factor, which accounted for the most variance across tasks. The complex-span tasks loaded heavily on the second factor. The antisaccade and sensory discrimination tasks tended to load onto the third factor; however, the antisaccade and pitch discrimination also loaded onto the first factor. The Flanker and Stroop tasks did not load well onto any factor. The two-factor model was very similar except that the fluid intelligence and working memory tasks loaded onto a single factor.
Next, we conducted a confirmatory factor analysis with four factors (see Fig. 4). The model was a good fit, $\chi^{2}(59)=$ $99.57, p<.05, C F I=.97, R M S E A=.05[.03, .06]$. The correlations among the four constructs were all high. Sensory discrimination correlated with the other cognitive constructs in a range of $r=.70-.90$. This model is consistent with what has been found with other modern studies of sensory discrimination; that sensory-discrimination ability is highly correlated with fluid intelligence and working-memory capacity (Deary

Table 3 Study 1 correlation matrix

\begin{tabular}{|c|c|c|c|c|c|c|c|c|c|c|c|c|c|}
\hline & RAPM & LetterSets & NumberSeries & OSpan & SymSpan & RotSpan & Antisaccade & Flanker & Stroop & Pitch & Loud & Line & Circle \\
\hline RAPM & $(.81)$ & & & & & & & & & & & & \\
\hline LetterSets & 0.56 & $(.87)$ & & & & & & & & & & & \\
\hline NumberSeries & 0.65 & 0.62 & $(.86)$ & & & & & & & & & & \\
\hline OSpan & 0.46 & 0.49 & 0.50 & $(.77)$ & & & & & & & & & \\
\hline SymSpan & 0.51 & 0.46 & 0.42 & 0.56 & $(.82)$ & & & & & & & & \\
\hline RatSpan & 0.61 & 0.49 & 0.48 & 0.55 & 0.69 & $(.84)$ & & & & & & & \\
\hline Antisaccade & 0.51 & 0.39 & 0.39 & 0.31 & 0.40 & 0.47 & $(.92)$ & & & & & & \\
\hline Flanker & -0.33 & -0.18 & -0.23 & -0.18 & -0.25 & -0.17 & -0.30 & $(.83)$ & & & & & \\
\hline Stroop & -0.23 & -0.19 & -0.20 & -0.15 & -0.19 & -0.21 & -0.17 & 0.13 & $(.75)$ & & & & \\
\hline Pitch & -0.53 & -0.45 & -0.49 & -0.37 & -0.38 & -0.41 & -0.46 & 0.26 & 0.18 & $(.96)$ & & & \\
\hline Loud & -0.34 & -0.31 & -0.27 & -0.28 & -0.24 & -0.38 & -0.40 & 0.16 & 0.19 & 0.42 & $(.86)$ & & \\
\hline Line & -0.26 & -0.21 & -0.18 & -0.21 & -0.18 & -0.29 & -0.25 & 0.12 & -0.00 & 0.20 & 0.32 & $(.54)$ & \\
\hline Circle & -0.16 & -0.16 & -0.12 & -0.16 & -0.16 & -0.19 & -0.14 & 0.11 & -0.02 & 0.16 & 0.27 & 0.15 & $(.86)$ \\
\hline
\end{tabular}

Computed correlation used pearson-method with pairwise-deletion. Split-half reliabilities are reported along the diagonal. Sensory discrimination task reliabilities were computed using Cronbach's alpha. Correlation in grey are non-significant, $p>.05$ 
Table 4 Exploratory factor analysis - rotated factor loadings

\begin{tabular}{llll}
\hline & Factor 1 & Factor 2 & Factor 3 \\
\hline RAPM & $\mathbf{0 . 6 7}$ & 0.35 & 0.29 \\
LetterSets & $\mathbf{0 . 6 0}$ & 0.34 & 0.19 \\
NumberSeries & $\mathbf{0 . 7 9}$ & 0.26 & 0.10 \\
SymSpan & 0.30 & $\mathbf{0 . 7 5}$ & 0.19 \\
OSpan & 0.40 & $\mathbf{0 . 5 3}$ & 0.18 \\
RotSpan & 0.33 & $\mathbf{0 . 7 0}$ & 0.36 \\
Antisaccade & $\mathbf{0 . 4 1}$ & 0.23 & $\mathbf{0 . 4 4}$ \\
Flanker & -0.28 & -0.10 & -0.19 \\
Stroop & -0.22 & -0.13 & -0.10 \\
Pitch discrimination & $\mathbf{- 0 . 5 3}$ & -0.17 & $\mathbf{- 0 . 4 0}$ \\
Loudness discrimination & -0.21 & -0.09 & $\mathbf{- 0 . 7 0}$ \\
Line discrimination & -0.12 & -0.13 & $\mathbf{- 0 . 3 9}$ \\
Circle discrimination & -0.06 & -0.10 & $\mathbf{- 0 . 3 1}$ \\
\hline
\end{tabular}

Note. Loadings $<.30$ are presented in gray. For each task, the loading in bold font is the factor(s) the task preferred to load onto

et al., 2004; Meyer et al., 2010; Troche et al., 2014). Our model provides evidence that sensory discrimination also correlates highly with attention control.

For each of the latent constructs, we tested whether we could restrict the correlation with sensory discrimination to $r$ $=1$ (Table 5). It did not impair model fit to restrict the correlation between sensory discrimination and attention control to $r=1$. However, it did impair model fit if we restricted the correlation between sensory discrimination and fluid intelligence or working-memory capacity to $r=1$. This suggests that all of the variance common amongst the individual sensory discrimination tasks (line, circle, loud, and pitch) and attention control tasks can be accounted for by a single factor. This is consistent with the exploratory factor analysis.

\section{Mediation models}

Our hypothesis was that attention control can account for relationship that sensory discrimination shares with fluid intelligence and working-memory capacity. To test this hypothesis, we conducted mediation analyses with structural equation models. Following the advice of Baron and Kenny (1986) on testing for mediation, we first examined the total relationship of fluid intelligence to sensory discrimination (without attention control). Then we included attention control as a mediator to test whether the total relationship from fluid intelligence to sensory discrimination could be fully or partially accounted for by attention control. We did the same thing replacing fluid intelligence with working-memory capacity. These models are presented in Fig. 5 and Fig. 6.

Fluid intelligence predicted statistically significant and substantial variance $(62.4 \%)$ in sensory discrimination (Fig. $5 a)$. When attention control was included as a mediator, fluid intelligence no longer directly predicted statistically significant variance in sensory discrimination (Fig. 5b). Next, we compared the mediation model in Fig. 5b with a model in which the direct path value from fluid intelligence to sensory discrimination is set to 0 ; in other words, a model comparison testing for full mediation versus partial mediation. The model comparison provided evidence of full mediation, it did not hurt model fit to restrict the direct path value to $0\left[\Delta \chi^{2}(1)=\right.$ $.38, p>.05]$. Attention control, therefore, fully mediated the relationship between fluid intelligence and sensory discrimination.

Working memory capacity predicted statistically significant and substantial variance $(47.6 \%)$ in sensory discrimination (Fig. 6a). When attention control was included as a mediator, working-memory capacity no longer directly predicted statistically significant variance in sensory discrimination (Fig. 6b). Model comparison provided evidence of full mediation, it did not hurt model fit to restrict the direct path value to $0\left[\Delta \chi^{2}(1)=.51, p>.05\right]$. Attention control, therefore, fully mediated the relationship between working-memory capacity and sensory discrimination.

\section{Discussion}

In Study 1, we showed that attention control has a strong relationship to sensory discrimination. In fact, the confirmatory factor analyses suggest that this relationship is at or near unity. This result suggests that the reason performance on one sensory discrimination task (i.e., pitch) correlates with performance on another sensory discrimination task (i.e., line length) can be completely explained by attention control. We did not find that fluid intelligence or working-memory capacity correlated with sensory discrimination at unity. Attention control was also able to fully mediate the relationship between fluid intelligence/working-memory capacity and sensory discrimination.

One potential limitation of our study is that our visual discrimination tasks had poor loadings, leading our sensorydiscrimination factor to be biased towards auditory discrimination. There are many possible explanations for why the visual discrimination tasks had poor loadings. It could be the case that auditory discrimination more strongly correlates with cognitive ability, but this interpretation is not consistent with previous studies (Deary et al., 2004). It could also be because the way we presented the visual and auditory stimuli were inherently different. For the visual stimuli, both were presented simultaneously on the screen, but we presented the auditory stimuli sequentially with a delay between the two stimuli. Sequential presentation increases the need for subjects to attend to the stimuli because the representation of both events must be maintained in order for them to be compared. For the simultaneous presentation of our visual stimuli, subjects did 


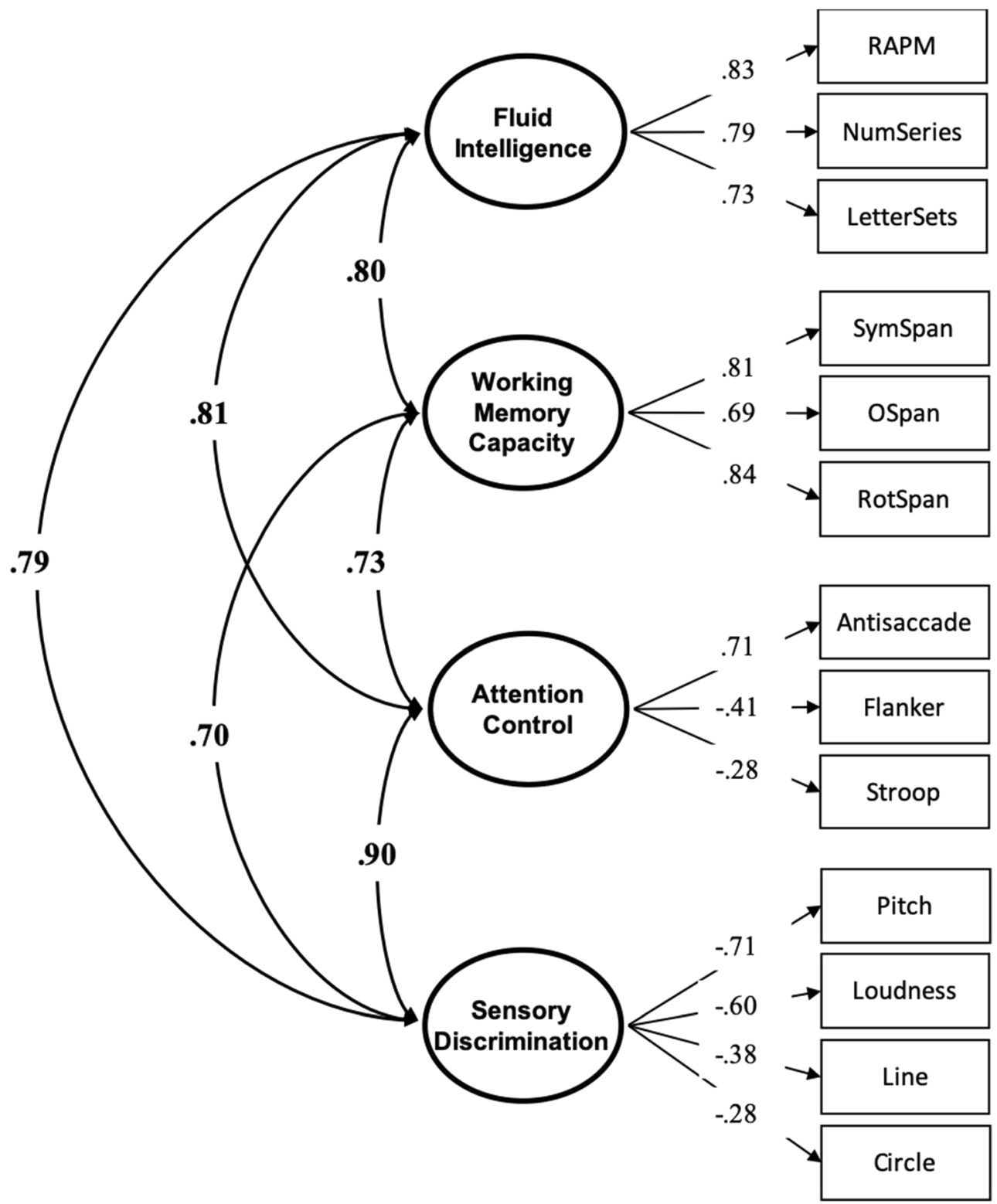

$$
\chi^{2}(59)=99.57, p<.05, \mathrm{CFI}=.97, \mathrm{RMSEA}[95 \% \mathrm{CI}]=.05[.03, .06]
$$

Fig. 4 Confirmatory factor analysis of full measurement model. The numbers to the left of each task are the task loading value onto the factor. The model was an excellent fit and all latent factors correlated highly with one another. $N=331$

Table 5 Model comparisons restricting the correlation of each factor with sensory discrimination to $r=1.0$

\begin{tabular}{llllll}
\hline Model $^{1}$ & $\chi^{2}$ & df & $\Delta \chi^{2}$ & $\Delta$ df & $p$ \\
\hline Attention control & 102.20 & 62 & 2.63 & 3 & .45 \\
Fluid intelligence & 131.33 & 62 & 31.76 & 3 & $<.001$ \\
Working-memory capacity & 163.54 & 62 & 69.97 & 3 & $<.001$ \\
No restrictions & 99.57 & 59 & & & \\
\hline
\end{tabular}

${ }^{1}$ Each of these restricted models are compared to the model in which all correlations are freely estimated (no restrictions). Non-significant pvalues indicate that the restricted model is a better fit (there is no improvement in model fit to estimate more parameters) not have temporal constraints on attending to the stimuli to be discriminated.

Although the low loadings of the visual discrimination tasks could be considered a limitation, it can also provide greater support for our findings. The reason for this is that the attention control tasks are all completely visual tasks. Our results show that the attention control based on visual attention tasks correlates at or near unity with a dominantly auditory sensory-discrimination factor. This suggests that our results are not due to domain-specific effects in any sensory modality but are domain general. 
a

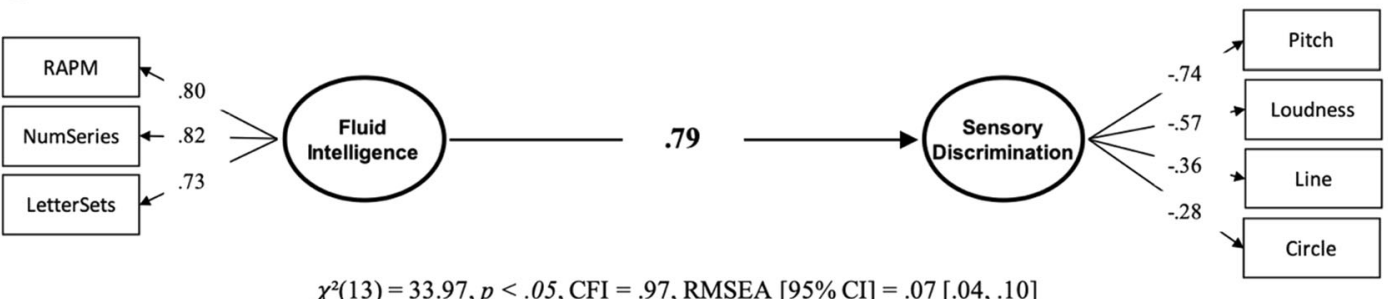

$\chi^{2}(13)=33.97, p<.05, \mathrm{CFI}=.97, \mathrm{RMSEA}[95 \% \mathrm{CI}]=.07[.04, .10]$

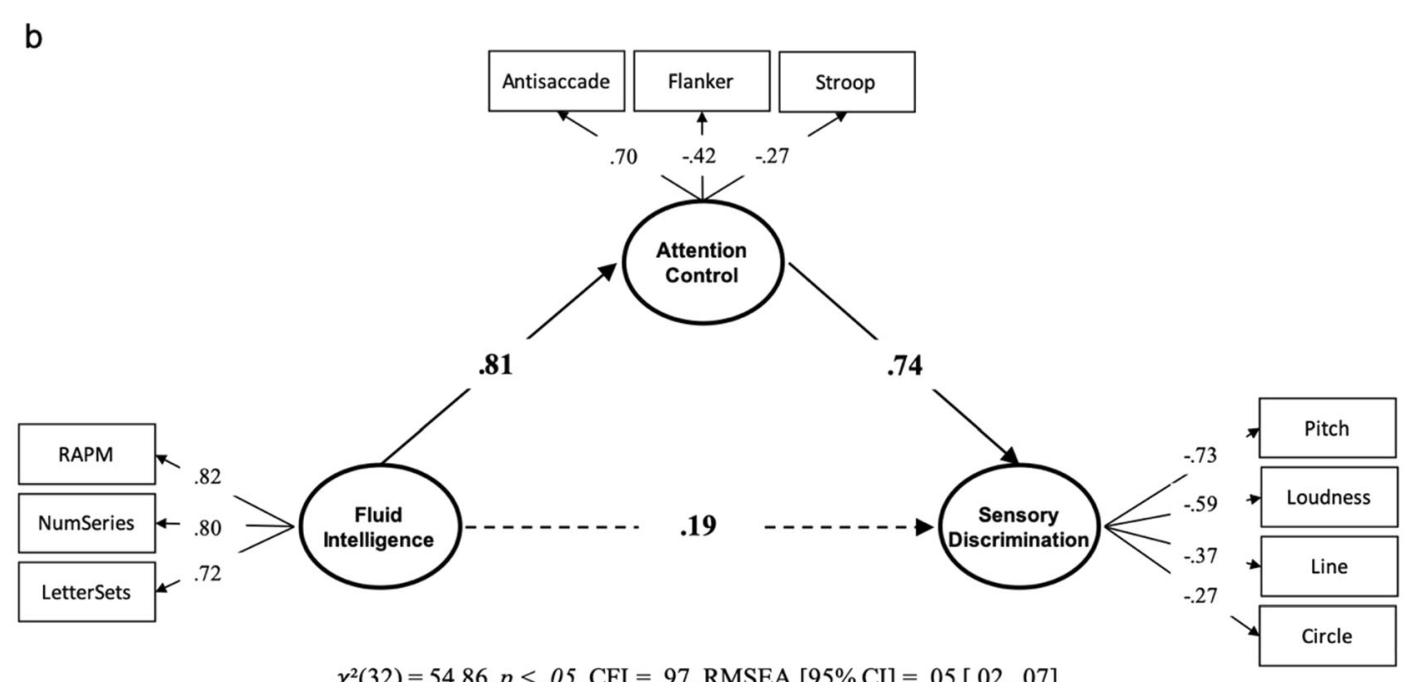

$\chi^{2}(32)=54.86, p<.05, \mathrm{CFI}=.97, \mathrm{RMSEA}[95 \% \mathrm{CI}]=.05[.02, .07]$

Fig. 5 Structural equation models of the Fluid Intelligence predicting Sensory Discrimination (a) without Attention Control and (b) with Attention Control as a mediator. Attention control fully mediated the relationship between fluid intelligence and sensory discrimination. $N=331$

Another potential limitation was the low loading of the Flanker and Stroop scores on the attention control factor in comparison to the high loading of antisaccade. This biases the attention control factor towards a single task, and it can be argued that what we have is more of an antisaccade factor then a general attention control factor (Draheim et al., 2019, 2020). Ideally, the magnitude for factor loadings should be greater than .35 (Bryne, 1994). The loadings for the Flanker and Stroop scores on attention control were somewhat typical for our lab but lower than what we ideally would like to see.

\section{Study 2}

The primary purpose of Study 2 was to replicate the findings in Study 1 while addressing some measurement issues with attention control. We examined the relationship between sensory-discrimination ability and attention control when using tasks that do not rely on reaction time, and specifically reaction-time difference scores (for a more detailed review of problems with reaction-time difference scores, see Draheim, Mashburn, Martin, \& Engle, 2019). First, it is a statistical property for difference scores to have lower reliability than their component scores (see Fig. 1 in Draheim et al., 2019). Second, even within young adults, individual differences in the emphasis of speed versus accuracy can result in reactiontime variables that are problematic and misleading (Draheim et al., 2016; Hughes et al., 2014; Vandierendonck, 2018). Reliance on reaction-time difference scores are prevalent in the measurement of attention control and executive functions and, as such, produce scores of demonstrably low reliability (Draheim et al., 2016; Friedman \& Miyake, 2004; Hedge et al., 2018; Hughes et al., 2014; Miyake et al., 2000; Oberauer et al., 2003; Paap \& Oliver, 2016; Rey-Mermet et al., 2018) and weaker relationships to other cognitive measures than predicted by theory (Draheim et al., 2019). Our lab's attention control factor typically consists of accuracy rates on antisaccade trials, the color Stroop reaction timeinterference effect, and the arrow Flanker reaction timeinterference effect. Because of these aforementioned issues with reaction time difference scores, this factor is dominated by performance on the antisaccade. ${ }^{3}$ As a result, the bulk of reliable variance in this attention control factor is primarily variance in the performance of the antisaccade task (see the

\footnotetext{
${ }^{3}$ In their Appendix B, Rey-Mermet, Gade, and Oberauer (2018) present a table of 23 experiments/studies assessing inhibition (what we call attention control) at the latent level. As shown in the table, a common finding among multiple labs is that one task "dominates" the construct such that it has very high loadings, whereas the other tasks have low loadings. In many cases, the dominant task was the antisaccade.
} 
a

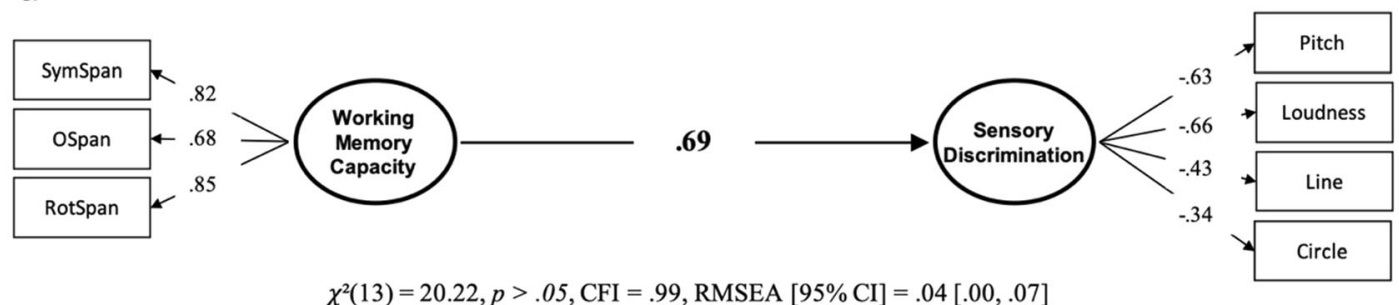

$\chi^{2}(13)=20.22, p>.05, \mathrm{CFI}=.99, \operatorname{RMSEA}[95 \% \mathrm{CI}]=.04[.00, .07]$

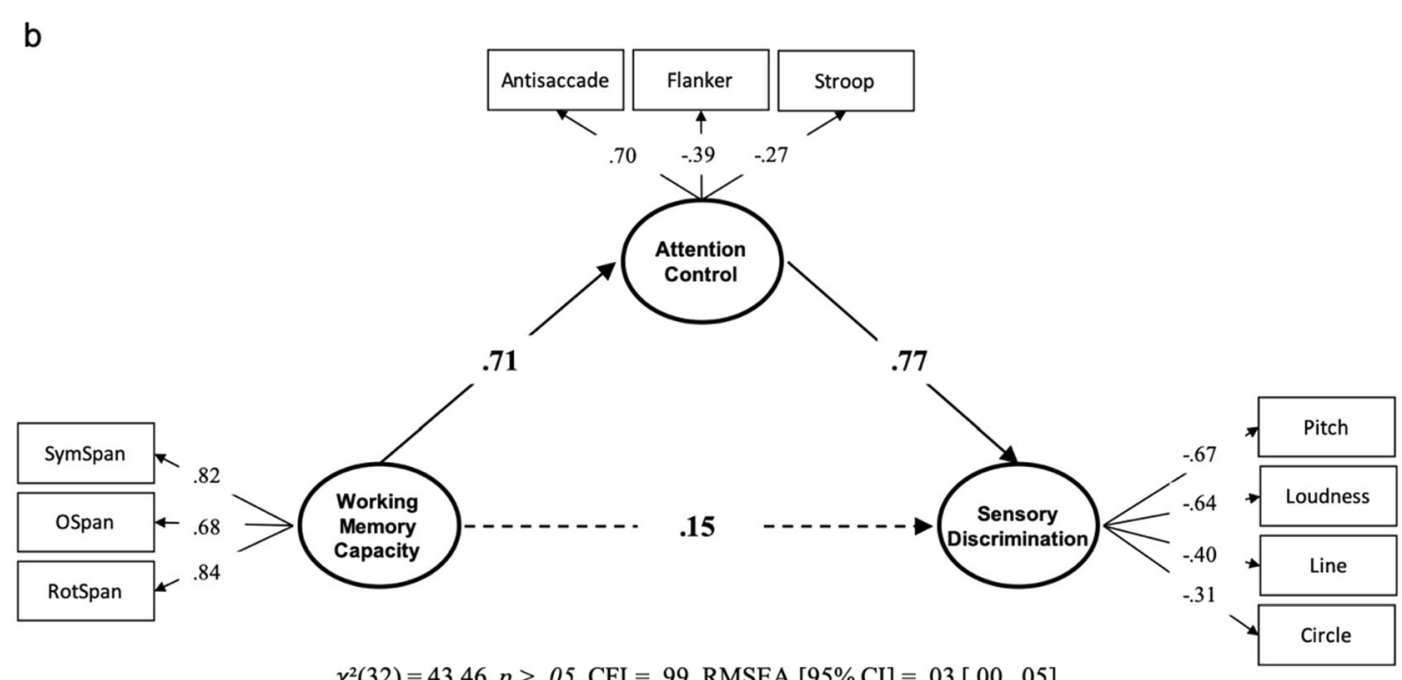

$\chi^{2}(32)=43.46, p>.05, \mathrm{CFI}=.99, \mathrm{RMSEA}[95 \% \mathrm{CI}]=.03[.00, .05]$

Fig. 6 Structural equation models of the Working-Memory Capacity predicting Sensory Discrimination (a) without Attention Control and (b) with Attention Control as a mediator. Attention control fully mediated

correlations involving these tasks in Table 3; also see the poor factor loadings for the Stroop and Flanker as opposed to the antisaccade in Fig. 4). Therefore, we wanted to improve the validity of our attention control factor by using tasks that do not rely on reaction time or difference scores and instead rely only on accuracy.

One potential criticism of not using tasks that rely on difference scores is that they are not process pure. Ignoring the low reliability of difference scores, this would be a fair point if subtraction methodology is a necessary condition of process purity - however it is not. In fact, researchers are aware that reaction-time difference scores are not process pure; they may be contaminated with speed-accuracy interactions and processing speed ability (J. Miller \& Ulrich, 2013; Rey-Mermet et al., 2018, 2019). The use of difference scores to isolate cognitive mechanisms are appropriate with experimental designs but are largely unreliable for individual differences research (Hedge et al., 2018). In addition, difference scores are designed to control for other processes at the within-subject level and this control does not transfer to the between-subject level (i.e., individual differences). For instance, a withinsubjects factor such as motivation (or insert any other factor or cognitive process individuals can vary on) might be controlled for between two conditions (e.g., congruent and the relationship between working-memory capacity and sensory discrimination. $N=331$

incongruent) but this does not mean it is controlled for at the between-subject level. Our approach to isolate cognitive mechanisms in individual differences research is to use latent variable analyses, such as structural equation modelling (Engle \& Martin, 2018). We discuss our approach to dealing with confounding factors in more detail in the General discussion.

This second study was part of a larger study in which we explored whether we could develop new tasks that are more reliable than the reaction-time difference score versions, which could potentially allow us to more validly measure individual differences in attention control. We report the reliability and validity results of the larger study elsewhere (Draheim et al. 2020; available at https://psyarxiv.com/ q985d/). Based on the larger study, the best three attention control tasks were the antisaccade, selective visual arrays with orientation judgment, and a newly developed task called the sustained attention-to-cue task. These tasks were selected based on results from Draheim et al. (2020), independently of their relationship to sensory discrimination (the sensory discrimination tasks were not analyzed in Draheim et al., 2020).

In this study, we are able to compare our findings using both our traditional reaction-time difference score versions 
and our new tasks. It has been suggested that after accounting for general processing speed, there is no unitary attention control construct; in other words, individual differences in attention control are really differences in processing speed (ReyMermet et al., 2018, 2019). We will also be able to assess the role of processing speed in these tasks and the relationship between attention control and sensory discrimination; and therefore, provide evidence of divergent validity between attention control and processing speed.

Another difference between Study 2 and Study 1 is the use of only auditory discrimination tasks. In Study 2, we used the loudness and pitch discrimination tasks from Study 1 and added a tone duration discrimination. Although this makes the sensory-discrimination factor less "general," this can be viewed as a slightly more stringent test of the relation between attention control and sensory discrimination. This is because the attention control tasks rely heavily on visual and oculomotor processes, whereas the sensory discrimination tasks depend on auditory processes. Therefore, any relation between attention control and sensory-discrimination ability cannot be attributed to domain-specific processes in one sensory modality. Finally, we also included a test-retest reliability of the sensory discrimination tasks.

\section{Method}

\section{Subjects}

The study was conducted at the Georgia Institute of Technology in Atlanta, GA, USA. The study consisted of four 2-hour sessions. All subjects were required to be native English speakers (i.e., learned English before the age of 5 years), 18-35 years of age, and not to have participated in a study with our lab before. No screening on vision or hearing was performed. Subjects were compensated with an average of $\$ 35$ on each session. There was also an option for Georgia Tech students to receive $2 \mathrm{~h}$ of course credits instead of monetary compensation on each session. This study was approved by the Georgia Institute of Technology's Institutional Review Board under Protocol H16409.

The data analyzed in this study were part of a larger data collection sample. The following link has a summary of the larger data collection procedure and a reference list of all publications to come out of this data collection sample with information on which tasks were used for each publication: https://osf.io/s5kxb/.

A total of 403 subjects completed the entire study and 11 subjects were removed due to having excessive missing data, for a final sample size of 392. Monte-Carlo simulations suggest that for stable estimates of correlations sample size should approach 250 (Schönbrodt \& Perugini, 2013). A subject may have missing data on a task for a variety of reasons including lost data file, task crashed before they could finish, or they did not meet a certain criterion of performance. The criteria of performance are defined on a task-by-task basis and are described in more detail for each task in the Scoring criteria section. Table 6 displays the demographics for the final 392 subjects.

\section{Tasks and procedures}

Subjects performed a battery of cognitive tasks in a group running room. The group running room had five subject stations and one research assistant monitored all subjects. The tasks were performed on a Windows 7 computer with an LED-backlit LCD monitor and subjects wore headphones during all tasks. The tasks were programmed in E-Prime 2.0.10 software (Psychology Software Tools, Inc. [E-Prime 2.0], 2016).

We measured working-memory capacity with the $a d$ vanced versions of the operation span, symmetry span, and rotation span tasks. The tasks to measure fluid intelligence were the Raven's advanced progressive matrices, letter sets, and number series. The procedures for the tasks used to measure working-memory capacity, fluid intelligence, Flanker, and Stroop were identical to those used in Study 1, and therefore will not be described here. However, in Study 2, 144 trials were administered for the Flanker and Stroop tasks (there were 216 and 486 trials in Study 1, respectively).

Attention control Based on our findings from the larger study, in which we investigated the reliability and validity of attention tasks that do not rely on difference scores, the three tasks that were ranked the highest across various measures of reliability and validity were the antisaccade, selective visual arrays, and the sustained attention to cue task (Draheim et al., 2020). The antisaccade and selective visual array tasks were already existing tasks used in the literature of attention and

Table 6 Subject demographics for Study $2(\mathrm{~N}=392)$

\begin{tabular}{ll}
\hline Demographic & Statistic \\
\hline Age (years) & Mean: 22 \\
& SD: 4.5 \\
Gender & Male: $39 \%$ \\
& Female: $60 \%$ \\
& Other: $1 \%$ \\
College student & Yes: $94.1 \%$ \\
& No: $5.9 \%$ \\
Ethnicity ${ }^{2}$ & White: $28.6 \%$ \\
& Black or African American: $31.2 \%$ \\
& Asian or Pacific Islander: 31.0\% \\
& Other: 9.2\% \\
\hline${ }^{1}$ College students included Georgia Tech and other colleges in Atlanta. \\
$47 \%$ of college students were from Georgia Tech \\
2 “Other" includes Native American, \\
Hispanic or Latino, Mix, and Other
\end{tabular}


working memory. The sustained attention to cue task was a new task that we developed.

Antisaccade. The procedures for the antisaccade are the same as in Study 1 with two exceptions. In Study 2, the antisaccade had 72 trials (48 trials in Study 1) and the task included eye tracking. Because the eye-tracking data are not reported here, the eye-tracking methods will not be described in detail. The eye-tracking procedure did require an initial calibration of the eye tracker with the subject's position before reading instructions for the task. No chinrest was used, but the subject was instructed to try to remain in the same seating position throughout the duration of the task. Other than that, there were no other demands on the subject due to the eye tracking.

Selective visual arrays with orientation judgment (VAorient-S). In this task subjects had to decide whether a probe array of stimuli was the same or different from the target array (Luck \& Vogel, 1997; Shipstead et al., 2014). The stimuli were red (RGB: 255, 0 0) and blue (RGB: 0, 0,255 ) rectangles in various orientations (horizontal, left diagonal, right diagonal, or vertical). Specifically, they had to make a judgment as to whether a single rectangle in the probe array had remained in the same orientation or was in a different orientation as the target array. They responded by pressing the 5 and 6 keys on the numpad, labeled "Yes" and "No" respectively. The trial sequence is presented in Fig. 7. Prior to each trial subjects were reminded to respond "Yes" for a same judgment and "No" for a different judgment and had to press the spacebar for the trial to begin. Thus, the task was selfpaced. After pressing the spacebar, there was a 1-s blank screen followed by a screen with a central fixation (+) for 1 s. After the fixation, a cue was presented, "RED" or "BLUE", to instruct the subject to attend to either red or blue rectangles; this was followed by a blank screen for $100 \mathrm{~ms}$. Next, a target array of blue and red rectangles differing in orientation (horizontal, left diagonal, right diagonal, or vertical) were presented for $250 \mathrm{~ms}$. After a delay (blank screen) of $900 \mathrm{~ms}$, a probe array with only the color of rectangles they were cued to attend to was presented with one of the rectangles highlighted by a white dot. The rectangle with the white dot changed orientation on $50 \%$ of the trials and remained the same on the other $50 \%$ of trials, while all other rectangles were identical to the target array. The probe array remained onscreen until a response was made. The subject had to make a response as to whether the rectangle remained in the same orientation, "Yes," or was in a different orientation, "No." The background color was set to "silver" (RGB: 192, 192, 192) and all words and fixation crosses were in black. The target array contained either five or seven rectangles per color (10 and 14 total), and a total of
48 trials were presented for each array set size. The dependent variable was a capacity score $(k)$, which is calculated using the single-probe correction (Cowan et al., 2005; Shipstead et al., 2014). This calculation is N* (hits + correction rejections -1 ), where $\mathrm{N}$ is the set size for that array. This calculation results in two separate $k$ scores, one for set size 5 and one for set size 7 , and the final dependent variable was the average $k$ for these two set sizes.

Sustained attention-to-cue task (SACT). In this task, subjects needed to sustain their attention on a visual circle cue presented at random locations on the screen and ultimately identify a target letter presented briefly at the center of the cue (Fig. 8). This task was designed as an accuracy version of the psychomotor vigilance task (Dinges \& Powell, 1985), with the addition of a distractor similar to that used in the antisaccade task. The stimuli for the task were presented against a gray background. Each trial started with a central black fixation. On half of the trials, the fixation was presented for $2 \mathrm{~s}$ and for the other half the fixation was presented for $3 \mathrm{~s}$. After the fixation, following a 300-ms tone, a large white circle cue was presented in a randomly determined location on either the left or right side of the screen. To orient the subject on the circle cue, the large circle began to immediately shrink in size until it reached a fixed size. Once the cue reached the fixed size, after a variable wait time (equally distributed amongst 2, 4, 8, and $12 \mathrm{~s}$ ), a white asterisk meant to serve as a distractor appeared at the center of the screen. The asterisk blinked on and off in 100-ms intervals for a total duration of $400 \mathrm{~ms}$. Then, a $3 \times 3$ array of letters was displayed at the center of the cue. The letters in the array consisted of B, D, P, and R. The central letter was the target letter and was presented in a dark gray font. The non-target letters were presented in black font with each letter occurring twice in the array and the target letter occurred three times. After $125 \mathrm{~ms}$ the central letter was masked with a \# for 1,000 ms. Only the central target letter was masked. After the mask, the response options were displayed in boxes horizontally across the upper half of the screen. The subject used the mouse to select whether the target was a B, D, P, or R. Feedback was given during the practice trials but not the experimental trials. Accuracy rate was the dependent variable.

Sensory discrimination To measure sensory discrimination, we used three auditory discrimination tasks. The procedures 


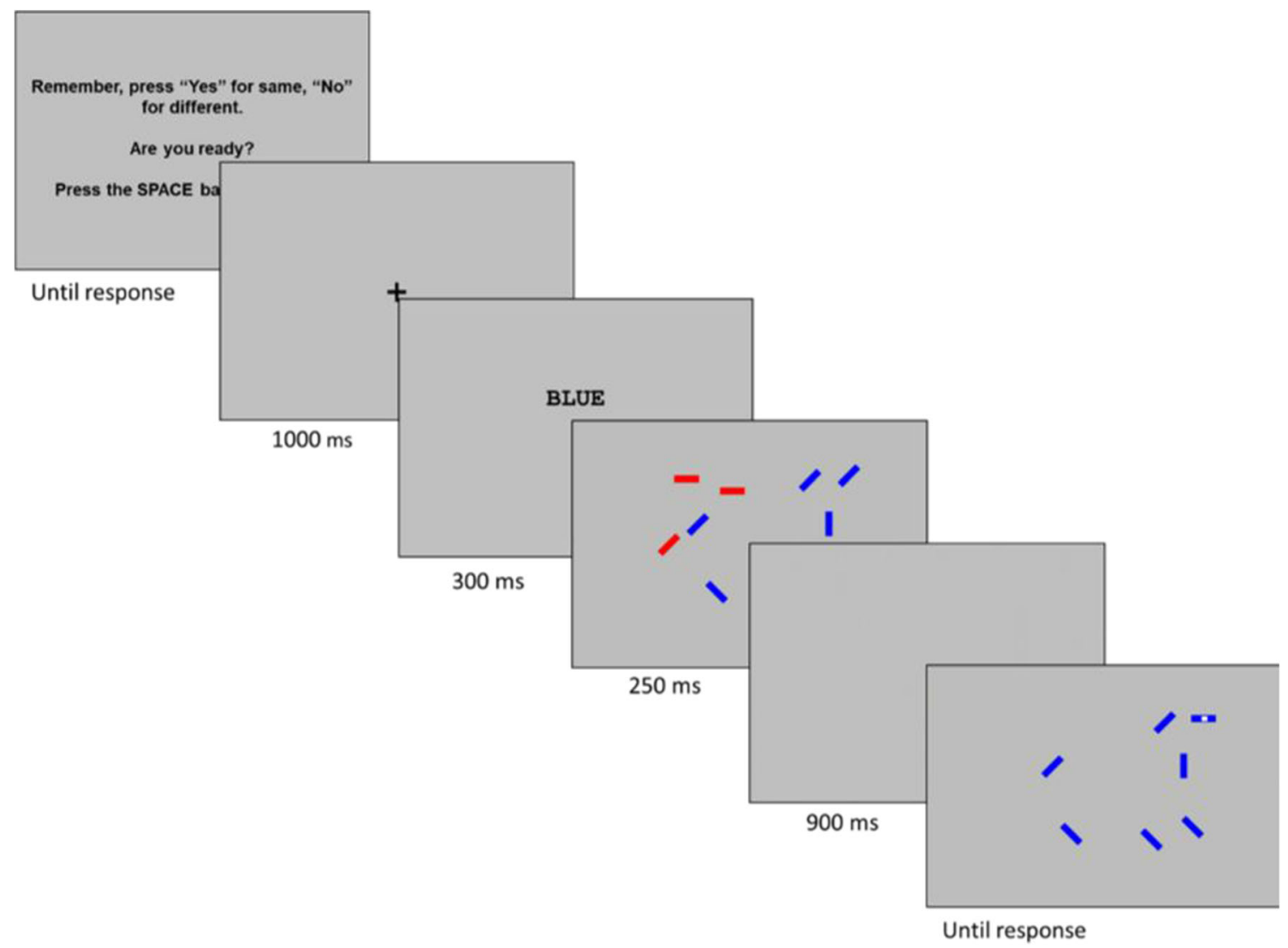

Fig. 7 Trial sequence for the selective visual arrays task

for the pitch and loudness discrimination tasks were identical to those used in Study 1 with the exception that the tasks included eye tracking. Because the eye-tracking data are not reported here, the eye-tracking methods will not be described in detail. The eye-tracking procedure did require an initial calibration of the eye tracker with the subject's position before reading instructions for the task. No chinrest was used, but the subject was instructed to try to remain in the same seating position throughout the duration of the task. Other than that, there were no other demands on the subject due to the eye tracking. The critical dependent measure on all sensory discrimination tasks is the average difference value of the last four reversals. As in Study 1, we conducted all the analyses using various dependent measures and overall the interpretation of the models was the same regardless of which estimate was used.

Duration discrimination. For this task subjects were presented with two auditory tones and indicated which tone was of longer duration. The two tones were presented with a $500-\mathrm{ms}$ interstimulus interval. After the tones had played, the subject was given unlimited time to decide which tone was of longer duration. All tones were pure and at $700 \mathrm{~Hz}$ and $60 \mathrm{~dB}$. All tones had a 5-ms fade- in at the beginning of the tone and a 5-ms fade-out at the end of the tone. For half of the trials the first tone had a longer duration and for the other half the second tone had a longer duration.

The first trial started at a tone duration difference of 100 $\mathrm{ms}$. For the first six trials the duration difference decreased (after correct trials) by $20 \mathrm{~ms}$ and increased (after incorrect trials) by $60 \mathrm{~ms}$. After the first six trials the duration difference decreased by $10 \mathrm{~ms}$ and increased by $30 \mathrm{~ms}$. The minimum duration difference was $10 \mathrm{~ms}$ and the maximum duration difference was $150 \mathrm{~ms}$. There was a total of 64 trials.

Processing speed A subset of subjects, $\mathrm{N}=176$, were recruited to come back for additional sessions in which they performed various tasks including parts of the Armed Services Vocational Aptitude Battery, multitasking tasks, and processing speed measures. Only the processing speed measures are relevant to this paper and therefore reported here. All processing speed measures were computerized versions of paper and pencil tests. In each case, subjects were instructed to respond as quickly and accurately as possible, but consistent with standard administration procedures, subjects were not alerted of the time limits of each task. 


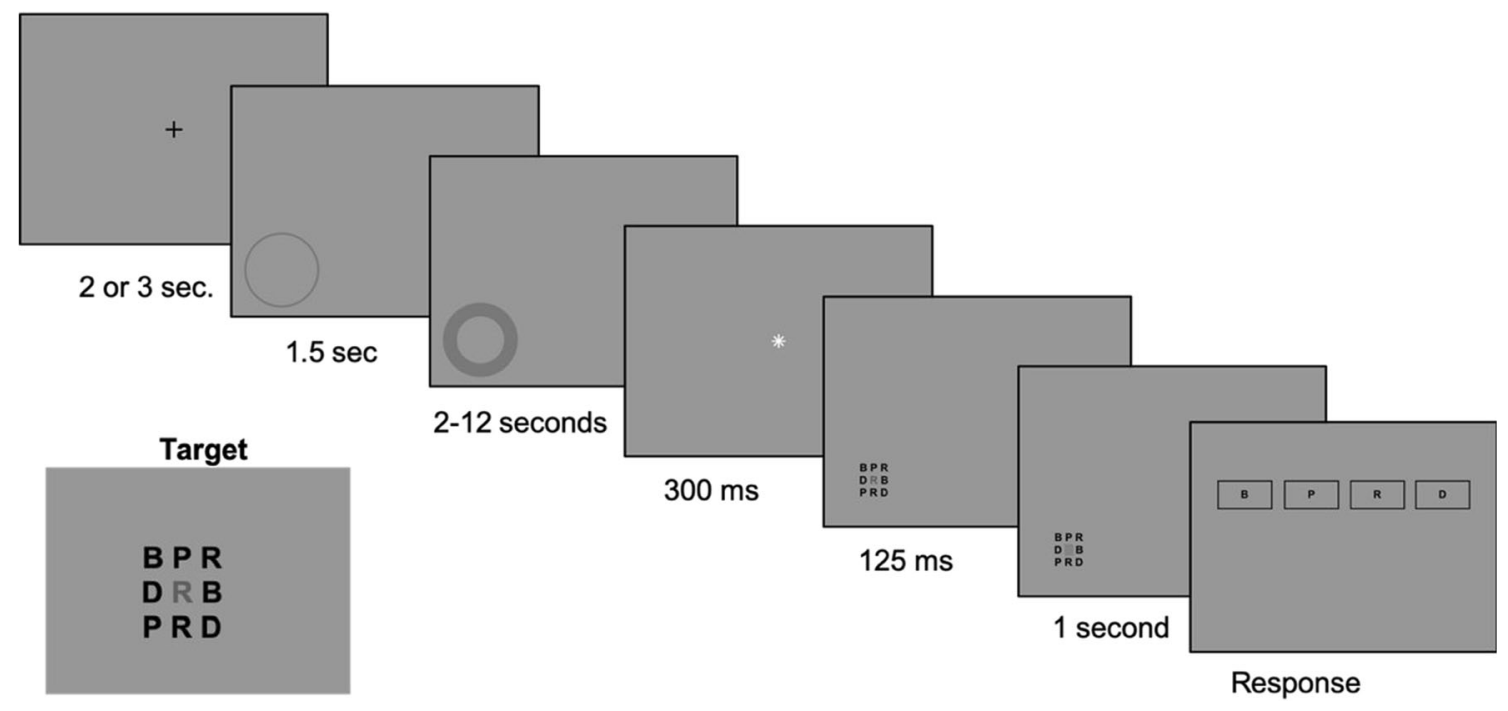

Fig. 8 Trial sequence for the sustained attention-to-cue task

Letter string comparison. In this version of the Letter String Comparison Task (Salthouse \& Babcock, 1991; Shipstead et al., 2014), subjects viewed strings of three, six, or nine consonants appearing to the left and right of a central line. The letter strings could either be the same or differ by a single letter. If different, the mismatching letter could appear in any location in the string. Subjects indicated their response by clicking on a button on the screen labeled SAME for identical strings or DIFF for mismatching strings. Letters were printed in white size 18 Courier New font on a black background. After completing six practice trials, subjects completed two 30-s blocks of the task. The dependent variable was the number of accurate responses across both blocks.

Digit string comparison. The Digit String Comparison task (Redick et al., 2012; Salthouse \& Babcock, 1991) was identical to the Letter String Comparison, except that subjects viewed and made judgments about strings containing three, six, or nine digits.

Digit symbol substitution. This adaptation of the Digit Symbol Substitution Task (Wechsler, 1997) has been modified to make it more amenable to computer administration and response collection via a standard number pad. The symbols used were the same as the paper-andpencil version of the task, and we endeavored to maintain the same basic demands. However, rather than viewing digits and reporting corresponding symbols, this task required subjects to view symbols and report the corresponding digits. On each trial, subjects were presented with two boxes juxtaposed one on top of the other in the center of the screen. A symbol appeared in the bottom box. Subjects were to consult a key presented at the top of the screen, and to indicate via key press with the digit that belonged in the top box. After ten practice trials, subjects completed $90 \mathrm{~s}$ of the task. The dependent variable was the number of correctly reported digits during that $90-\mathrm{s}$ period.

\section{Test-retest reliability}

As part of the large-scale study that this was a part of, we collected test-retest reliability on a number of attention control tasks and the sensory discrimination tasks. We will not present data from the attention control tasks as that is part of a larger study; more directly relevant to this study is the test-retest reliability of the sensory discrimination tasks. For the sensory discrimination tasks, we brought back a total of 63 subjects. The average time between administration of test-retests was 190 days.

\section{Data processing and analysis}

Scoring criteria The same scoring criteria were used as from Study 1 and will not be described in detail again. For the visual arrays 4 task, if the subjects mean accuracy was 3.5 SDs below the mean, then their $k$ score for that task was removed. Then for every task, we removed univariate outliers by removing task scores that were 3.5 SDs above or below the mean for that task. If a subject had missing or removed data on more than half of the task indicators for any one construct they were completely removed from any further analyses. After using these scoring criteria, a total of 11 subjects were removed from further analyses resulting in a final sample size of 392.

Data analysis We performed the exact same statistical analyses in Study 2 as we did in Study 1. All analyses were conducted in R statistical software (R Core Team, 2018). The R package lavaan (Rosseel, 2012) was used for all confirmatory factory analyses and structural equation models, treatment of 
missing values was set to full-information maximum likelihood. The same criteria for model fit in Study 1was used in Study 2.

\section{Results}

Data, analysis scripts, and results output are available via the Open Science Framework: https://osf.io/hsqru/. The descriptive statistics for the individual tasks are presented in Table 7. The task-level correlation matrix is presented in Table 8. Again, the sensory discrimination tasks correlated moderately with the other cognitive tasks, but slightly lower than in Study 1. The sensory discrimination tasks also showed high internal consistency; they tend to correlate more with each other than with the cognitive tasks.

First, we present structural equation models using the new attention control tasks as our primary analysis. We follow up this analysis with models using the same attention control construct used in Study 1. That is, we used a latent consisting of the antisaccade, and the reaction-time difference score versions of the Stroop and Flanker. We also present the test-retest reliability of the sensory discrimination tasks. Finally, we present some models controlling for processing speed.

\section{Measurement model}

First, we conducted an exploratory factor analysis to investigate the latent structure of the tasks in a data-driven manner.
We used principal axis factoring with a varimax rotation. Visual inspection of the scree plot indicated at least three factors. Three factors had eigenvalues greater than 1 . Therefore, we specified a model with four factors and a model with three factors. The four-factor model is presented in Table 9. The fluid intelligence tasks loaded heavily on the first factor, which accounted for the most variance across tasks. The complex-span tasks loaded heavily on the second factor. The sensory discrimination tasks and the SACT loaded onto the third factor. The antisaccade, visual arrays, and SACT tasks loaded onto the fourth factor. The three-factor model was similar except that the attention control and sensory discrimination tasks loaded onto a single factor. Therefore, the attention control and sensory discrimination tasks could either load onto a single factor or separate factors.

Next, we conducted a confirmatory factor analysis with four factors (see Fig. 9). The model was a good fit, $\chi^{2}(48)=$ $111.19, p<.05, C F I=.96$, RMSEA $=.06[.04, .07]$. Except for the correlation between working-memory capacity and sensory discrimination $(r=.41)$, the correlations among the four constructs were all high. Sensory discrimination correlated with the other cognitive constructs in a range of $r=.41$ .79. Overall, compared to Study 1 the latent variables shared less variance with each other.

For each of the latent constructs, we tested whether we could set the correlation with sensory discrimination to $r=1$ (Table 10). Model fit was impaired when the correlation of sensory discrimination with either attention control, fluid

Table 7 Descriptive statistics for each task in Study $2(\mathrm{~N}=392)$

\begin{tabular}{|c|c|c|c|c|c|}
\hline & Mean (SD) & Min-max & Skewness & Kurtosis & $\%$ Missing \\
\hline \multicolumn{6}{|l|}{$\mathbf{G f}(A C C)$} \\
\hline RAPM & $10.2(3.3)$ & $1-18$ & -0.46 & -0.16 & 0.00 \\
\hline LetterSets & $17.0(4.4)$ & $5-28$ & -0.33 & -0.28 & 0.51 \\
\hline NumberSeries & $9.8(3.1)$ & $2-15$ & -0.42 & -0.46 & 0.26 \\
\hline \multicolumn{6}{|l|}{ WMC (ACC) } \\
\hline SymSpan & $27.7(10.6)$ & $0-54$ & -0.10 & -0.39 & 1.79 \\
\hline OSpan & $55.4(15.9)$ & $4-82$ & -0.75 & 0.13 & 0.77 \\
\hline RotSpan & $24.3(9.4)$ & $0-49$ & -0.13 & -0.23 & 1.02 \\
\hline \multicolumn{6}{|l|}{ Attention } \\
\hline Antisaccade $(A C C)$ & $0.79(0.15)$ & $0.28-1.0$ & -0.87 & -0.01 & 2.30 \\
\hline SACT (ACC) & $0.70(0.19)$ & $0.16-1.0$ & -0.75 & -0.08 & 3.86 \\
\hline VAorient-S (k) & $1.86(1.17)$ & $0-5.32$ & 0.19 & -0.59 & 0.77 \\
\hline Flanker $(R T)$ & $80.2(44.2)$ & $-93.1-240.9$ & 0.89 & 2.28 & 3.83 \\
\hline Stroop $(R T)$ & $130.9(86.1)$ & $-81.8-452.9$ & 0.75 & 0.79 & 4.08 \\
\hline \multicolumn{6}{|l|}{ Discrimination } \\
\hline Pitch $(H z)$ & $13.3(6.8)$ & $2.5-23.5$ & 0.18 & -1.43 & 1.02 \\
\hline Loudness $(d B)$ & $4.9(2.5)$ & $2.5-12.5$ & 1.28 & 0.75 & 1.28 \\
\hline Duration $(m s)$ & $62.9(31.7)$ & $25-145$ & 0.96 & 0.03 & 2.30 \\
\hline
\end{tabular}

Note. $G f$ fluid intelligence, $W M C$ working-memory capacity, $A C C$ accuracy, $R T$ reaction time interference effect, $H z$, hertz, $d B$ decibels, $m s$ milliseconds Gf and WMC tasks were calculated as total correct. Antisaccade and SACT were calculated as proportion correct. VAorient-S was calculated as $k$ 


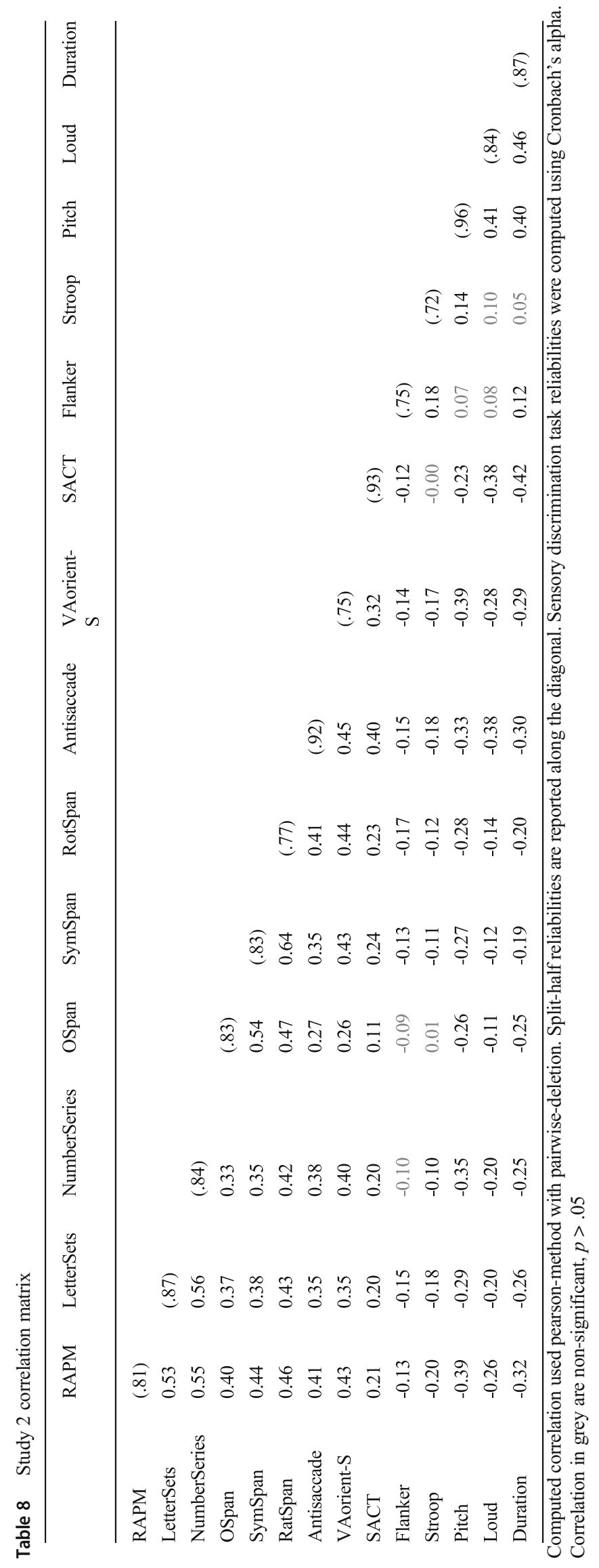


Table 9 Exploratory factor analysis - rotated factor loadings

\begin{tabular}{lllll}
\hline & Factor 1 & Factor 2 & Factor 3 & Factor 4 \\
\hline RAPM & $\mathbf{0 . 6 2}$ & 0.33 & 0.25 & 0.12 \\
LetterSets & $\mathbf{0 . 6 2}$ & 0.28 & 0.16 & 0.11 \\
NumberSeries & $\mathbf{0 . 7 2}$ & 0.20 & 0.15 & 0.16 \\
SymSpan & 0.20 & $\mathbf{0 . 7 8}$ & 0.08 & 0.25 \\
OSpan & 0.28 & $\mathbf{0 . 6 2}$ & 0.15 & -0.08 \\
RotSpan & 0.33 & $\mathbf{0 . 6 3}$ & 0.08 & 0.32 \\
Antisaccade & 0.30 & 0.21 & $\mathbf{0 . 3 6}$ & $\mathbf{0 . 4 6}$ \\
VAorient-S & $\mathbf{0 . 3 3}$ & 0.29 & 0.29 & $\mathbf{0 . 4 0}$ \\
SACT & 0.05 & 0.11 & $\mathbf{0 . 4 9}$ & $\mathbf{0 . 3 6}$ \\
Pitch Discrimination & -0.32 & -0.18 & $\mathbf{- 0 . 4 8}$ & -0.09 \\
Loudness Discrimination & -0.13 & -0.02 & $\mathbf{- 0 . 6 6}$ & -0.19 \\
Duration Discrimination & -0.16 & -0.14 & $\mathbf{- 0 . 7 0}$ & -0.01 \\
\hline
\end{tabular}

Note. Loadings $<.30$ are presented in gray. For each task, the loading in bold font is the factor(s) the task preferred to load onto

intelligence, or working-memory capacity was set to $r=1$. Unlike Study 1, in Study 2 attention control could not account for all the variance shared amongst the sensory discrimination tasks. However, sensory discrimination still correlated highly with attention control $(r=.79)$. The confirmatory factor analysis suggests that a four-factor model is preferred over a threefactor model, in which the attention control and sensory discrimination tasks load onto a single factor.

\section{Mediation model}

Again, we asked whether attention control can account for the relationship that sensory discrimination shares with fluid intelligence and working-memory capacity. To test this hypothesis, we conducted mediation analyses with structural equation models. Following the advice of Baron and Kenny (1986) on testing for mediation, we first examined the total relationship of fluid intelligence to sensory discrimination (without attention control). Then we included attention control as a mediator to test if the total relationship from fluid intelligence to sensory discrimination could be fully or partially accounted for by attention control. We did the same thing replacing fluid intelligence with working-memory capacity. These models are presented in Figs. 10 and 11.

Fluid intelligence predicted statistically significant variance $(36.0 \%)$ in sensory discrimination (Fig. 10a). When attention control was included as a mediator, fluid intelligence no longer directly predicted statistically significant variance in sensory discrimination (Fig. 10b). Next, we compared the mediation model in Fig. 10b with a model in which the direct path value from fluid intelligence to sensory discrimination is set to 0 ; in other words, a model comparison testing for full mediation versus partial mediation. The model comparison provided evidence of full mediation, it did not hurt model fit to restrict the direct path value to $0\left[\Delta \chi^{2}(1)=.04, p>.05\right]$. Attention control, therefore, fully mediated the relationship between fluid intelligence and sensory discrimination, replicating our findings from Study 1.

Working-memory capacity predicted statistically significant variance $(16.8 \%)$ in sensory discrimination (Fig. 11a). When attention control was included as a mediator, working-memory capacity no longer positively predicted variance in sensory discrimination but the relationship reversed to negative and statistically significant (Fig. 11b). Model comparison provided evidence that it did hurt model fit to restrict the direct path value to $0\left[\Delta \chi^{2}(1)=6.70, p<.05\right]$. These results are a bit odd and difficult to interpret. The total variance between working-memory capacity and sensory discrimination $(16.8 \%)$ was reduced to $7.8 \%$ when accounting for attention control - however, the direction of the relationship reversed. Therefore, the most we can conclude form this mod$\mathrm{el}$ is that attention control accounted for the positive relationship between working-memory capacity and sensory discrimination.

\section{Flanker and Stroop}

We conducted the same analyses using the reaction-time difference score versions of the Stroop and Flanker. In the confirmatory factor analysis (Fig. 12), for the most part, the latent correlations were equivalent to the models that used the new tasks instead. However, the correlation between attention control and sensory discrimination was slightly lower $(r=.71$ compared to $r=.79$ ). While the factor loadings were not problematic when the new tasks were used, the factor loadings for Flanker and Stroop were very poor in this model (Flanker $=-.26$, Stroop $=-.27$, Antisaccade $=.73$ ). This means most of the variance in the attention control factor was heavily biased towards the antisaccade task; the reaction-time difference score versions of the Flanker and Stroop contributed very little reliable variance. The structural equation models fully replicated what was found in Study 1. Attention control fully mediated the relationship of fluid intelligence/working-memory capacity to sensory discrimination (Figs. 13 and 14).

\section{Test-retest reliability}

To calculate the test-retest reliability of the sensory discrimination tasks we simply correlated the scores at the first administration with the scores at the second administration. The testretest reliabilities are presented in Table 11. Given that the average time between administrations was 6 months, exceedingly long for a typical test-retest study, these correlations represent a moderate to high reliability of the sensory discrimination tasks, especially the pitch discrimination task $(\mathrm{r}=.81)$. This provides even greater confidence that our findings over 


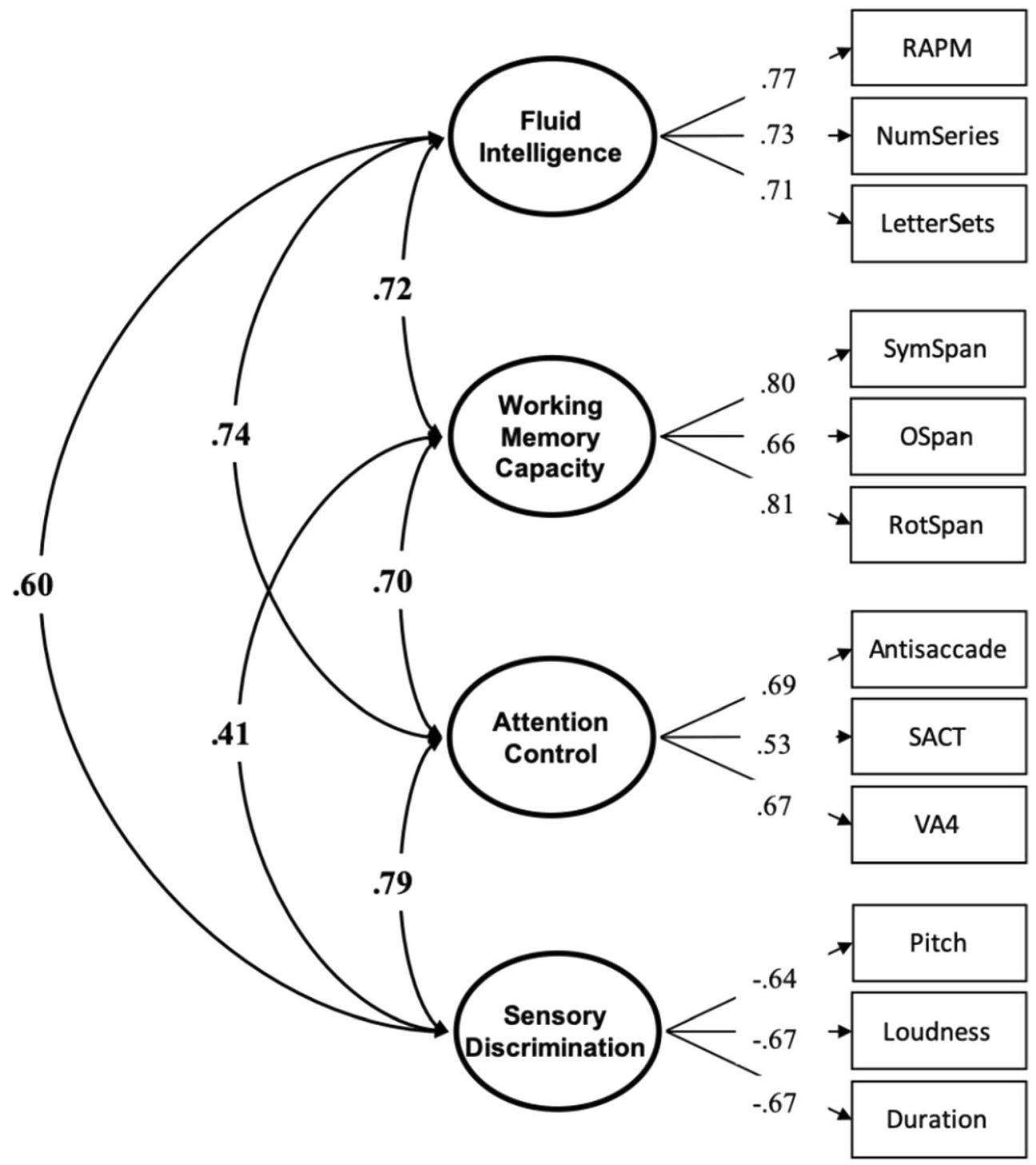

$$
\chi^{2}(48)=111.19, p<.05, \text { CFI }=.96, \text { RMSEA }[95 \% \mathrm{CI}]=.06[.04, .07]
$$

Fig. 9 Confirmatory factor analysis of full measurement model. The numbers to the left of each task were the task loading value onto the factor. The model was an excellent fit and all latent factors correlated

Table 10 Model comparisons setting the correlation of each factor with sensory discrimination to $r=1.0$

\begin{tabular}{llllll}
\hline Model $^{1}$ & $\chi^{2}$ & df & $\Delta \chi^{2}$ & $\Delta$ df & $p$ \\
\hline Attention control & 156.11 & 51 & 44.92 & 3 & $<.001$ \\
Fluid intelligence & 231.34 & 51 & 120.15 & 3 & $<.001$ \\
Working-memory capacity & 299.32 & 51 & 188.14 & 3 & $<.001$ \\
No restrictions & 116.96 & 48 & & & \\
\hline
\end{tabular}

${ }^{1}$ Each of these restricted models are compared to the model in which all correlations are freely estimated (no restrictions). Non-significant pvalues indicate that the restricted model is a better fit (there is no improvement in model fit to estimate more parameters) highly with one another, with the exception of Working Memory Capacity and Sensory Discrimination. $N=392$

Studies 1 and 2 are moderately to highly reliable and stable over time.

\section{Processing speed}

Processing speed may potentially be a confounding factor, in that it has been suggested that processing speed is the reason attention control tasks correlate with one another (ReyMermet et al., 2018, 2019). Therefore, it is important to test whether processing speed can account for our findings. We were able to test this in a smaller sub-sample of $\mathrm{N}=176$. The confirmatory factory analysis (Fig. 15) showed that attention control and sensory discrimination are still highly correlated 
a

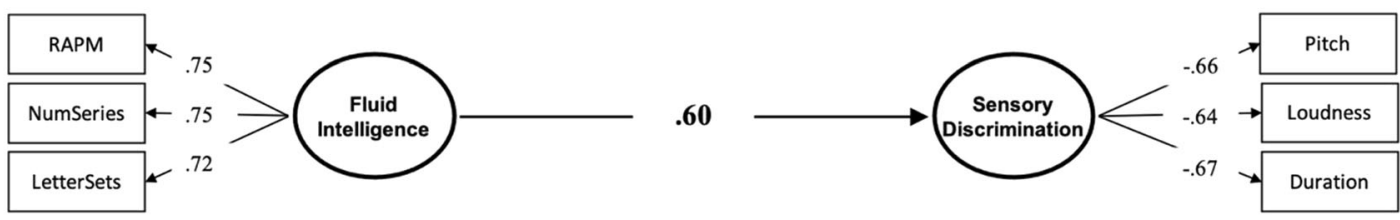

$\chi^{2}(8)=17.60, p<.05, \mathrm{CFI}=.98, \mathrm{RMSEA}[95 \% \mathrm{CI}]=.06[.02, .09]$

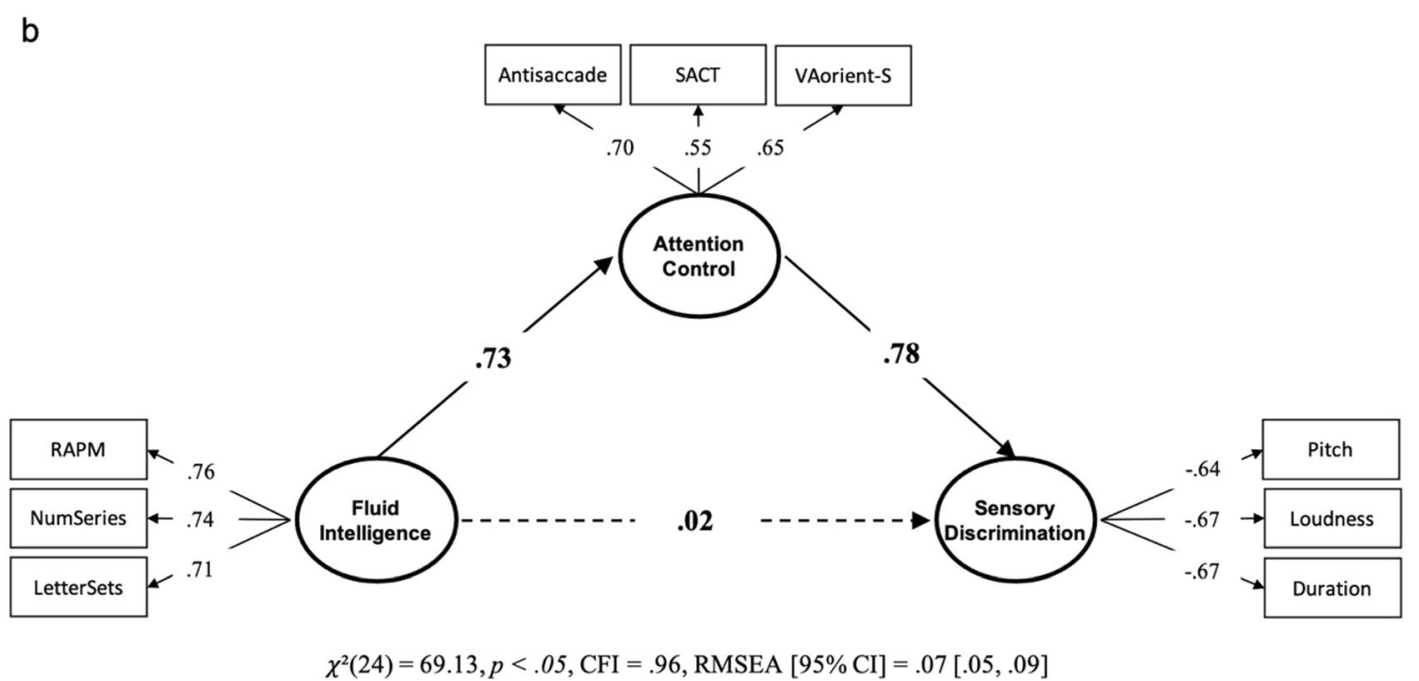

Fig. 10 Structural equation models of the Fluid Intelligence predicting Sensory Discrimination (a) without Attention Control and (b) with Attention Control as a mediator. Attention control fully mediated the relationship between fluid intelligence and sensory discrimination. $N=392$

in this sub-sample. Processing speed is also moderately correlated with both attention control and sensory discrimination. We tested whether processing speed can account for any variance between attention control and sensory discrimination by including it as a mediator (Fig. 16). It did not; processing speed did not account for any variance between attention control and sensory discrimination. The effect of attention control on sensory discrimination through processing speed was small and non-significant, $b=-.07, p>.05$. This provides some evidence that individual differences in processing speed cannot account for our findings.

To further test the role of processing speed in the attention control tasks, we extracted variance common to the processing speed measures out of the attention control tasks and again tested the relationships to sensory discrimination (Fig. 17). The model suggests that there is some variance in the attention control tasks that is common with the processing speed measures; however, attention control is still a stronger predictor of sensory discrimination. This is a rather strong test as the processing speed factor is now the more "general" factor as it has more tasks loading onto it. It likely contains more than just processing speed variance. The attention control factor, however, now has less variance and is the less "general" factor; yet, it is still a stronger predictor.

\section{Discussion}

In Study 2, we again showed that attention control is an important construct in accounting for the relationship between sensory discrimination and fluid intelligence/workingmemory capacity. Overall, in Study 2 the correlations amongst all the latent factors were smaller than Study 1. In Study 1, the mean correlation amongst the latent factors was $r$ $=.79$, and in Study 2 it was $r=.66$. Unlike Study 1, we did not find that we could set the correlation between attention control and sensory discrimination to $r=1$; however, the correlation was still high $r=0.79$. This suggests that most of the variance shared among the sensory discrimination tasks can be accounted for by individual differences in attention control. As in Study 1, attention control fully mediated the relationship between fluid intelligence and sensory discrimination; however, the mediation with working-memory capacity was more complicated. Therefore, the main findings in Study 1 were for the most part replicated in Study 2. We were also able to show that processing speed cannot account for our findings. Whether we controlled for processing speed at the latent construct-level or task-level, processing speed was not able to account for the relationship between attention control and sensory discrimination. 
a

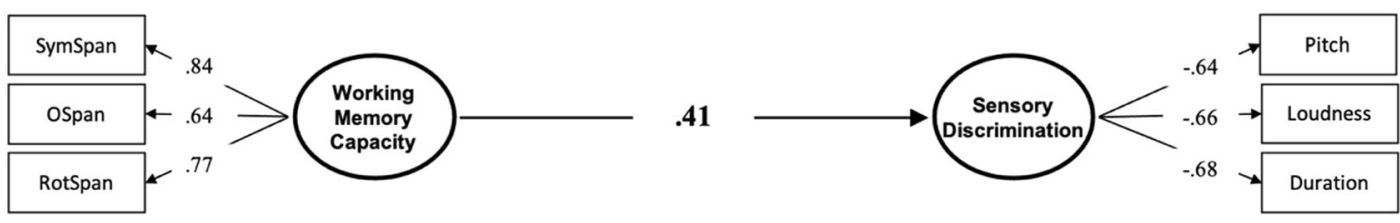

$\chi^{2}(8)=19.45, p<.05, \mathrm{CFI}=.98, \mathrm{RMSEA}[95 \% \mathrm{CI}]=.06[.03,1.0]$

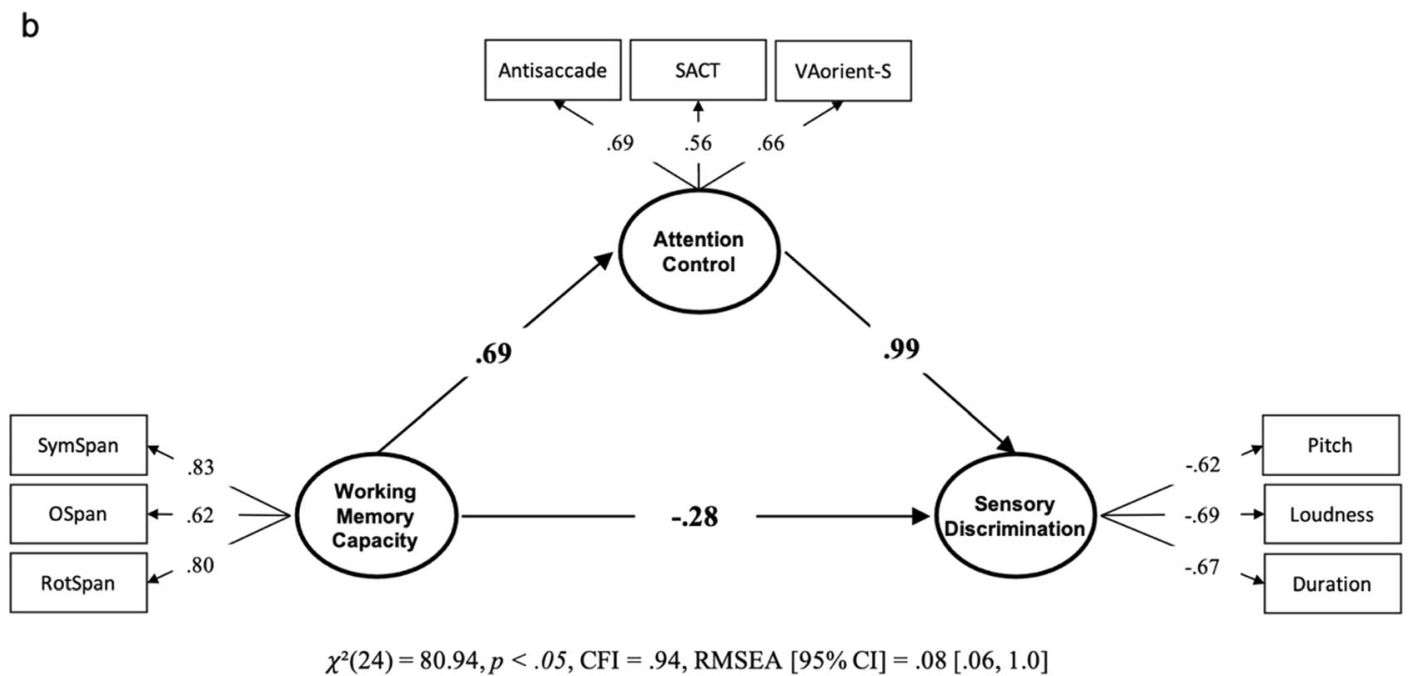

Fig. 11 Structural equation models of the Working-Memory Capacity predicting Sensory Discrimination (a) without Attention Control and (b) with Attention Control as a mediator. $N=392$

There are some important differences between the sample of subjects from Study 1 and those from Study 2. In Study 1, there was an even balance of college and non-college (community) subjects, whereas in Study 2 almost the entire sample was college students. This can potentially skew the distribution towards higher ability subjects. Although we recruited from a range of colleges and technical schools in Atlanta, about $50 \%$ of Study 2 subjects (vs. $24 \%$ in Study 1) were Georgia Tech students (a highly competitive engineering school). As such, there was a higher mean performance and smaller SD on every task in Study 2 compared to Study 1. These differences in subject samples may be one reason there were overall lower correlations amongst the latent factors in Study 2, and the reason attention control and sensory discrimination did not correlate at $r=1$.

Another difference between the two studies is that in Study 2 we used only auditory discrimination tasks, leading to a less "general" discrimination factor. Given that the attention control tasks all rely heavily on visual processing, our failure to replicate this finding from Study 1 may be due to one factor being more visual and the other more auditory. Despite these potential differences in sense modality between the two factors, we still find a high correlation between the two factors, $r$ $=.79$, and the model with the correlation set to $r=1$ is still a good fitting model.

\section{General discussion}

In two studies we have shown that attention control plays a prominent role in sensory discrimination. First, we found that the correlation between attention control and sensorydiscrimination ability is high; $r=0.79-.90$ (Fig. 4 and Fig. 9). Although there may be differences in domain-specific sense faculties, our results suggest any shared relations at the "general" or cross-domain level can largely be attributed to attention control processes. More importantly, we also found that attention control fully mediated the relationships of fluid intelligence and working-memory capacity to sensory discrimination (Figs. 5, 6, 10, and 11). We were also able to rule out the effects of processing speed in accounting for the relationship between attention control and sensory discrimination (Figs. 16 and 17). Based on the results from our two studies, we argue that attention control is the reason sensory discrimination correlates with fluid intelligence.

It could also be argued that on face value, whereas the attention control tasks require some degree of discrimination, the sensory discrimination tasks do not require distractor interference or response inhibition. Therefore, it could be that sensory discrimination is the more fundamental process that is common to the attention control and sensory discrimination tasks. Because our studies were correlational in nature, we 


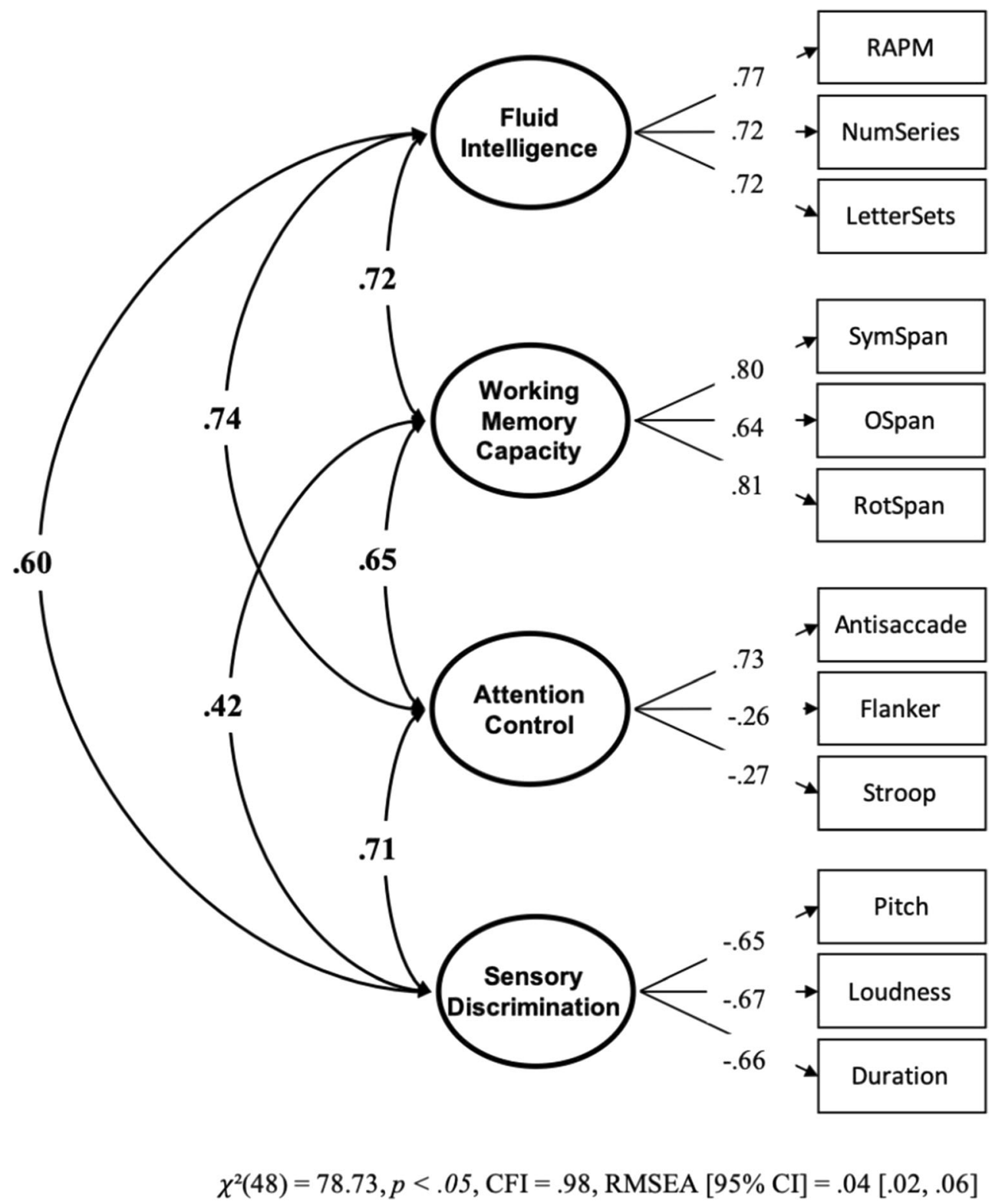

Fig. 12. Alternative confirmatory factor analysis of full measurement model in Study 2 with traditional Flanker and Stroop. $\mathrm{N}=392$

cannot determine causal direction. However, we can test whether attention control or sensory discrimination is more fundamental in accounting for variance associated with fluid intelligence. If sensory discrimination mediates the relationship between fluid intelligence and attention control, this would suggest that attention control is related to fluid intelligence only due to processes of sensory discrimination and the possibility that sensory discrimination is more fundamental is viable. If sensory discrimination does not mediate this relationship, then this would suggest that attention control is related to fluid intelligence above and beyond sensory discrimination. Therefore, not only would attention control fully explain why sensory discrimination correlates with fluid intelligence, but there is additional non-sensory discrimination related variance in attention control related to fluid intelligence.
This would provide discrimination validity to the interpretation that attention control is more fundamental to processes of sensory discrimination rather than vice versa.

The near perfect correlation between attention control and sensory discrimination, $r=.90$, in Study 1 is a limitation in terms of testing for this. If two variables correlate at $r=.90$, it is no surprise that they would also mediate each other's relationships with other variables. However, in Study 2, because attention control and sensory discrimination had a smaller but still high correlation, $r=.79$, we can test this model without the limitation from Study 1. Testing this model showed that sensory discrimination cannot fully mediate the relationship between attention control and fluid intelligence or workingmemory capacity (Figs. 18 and 19). In other words, sensory discrimination cannot account for the relationship of attention 


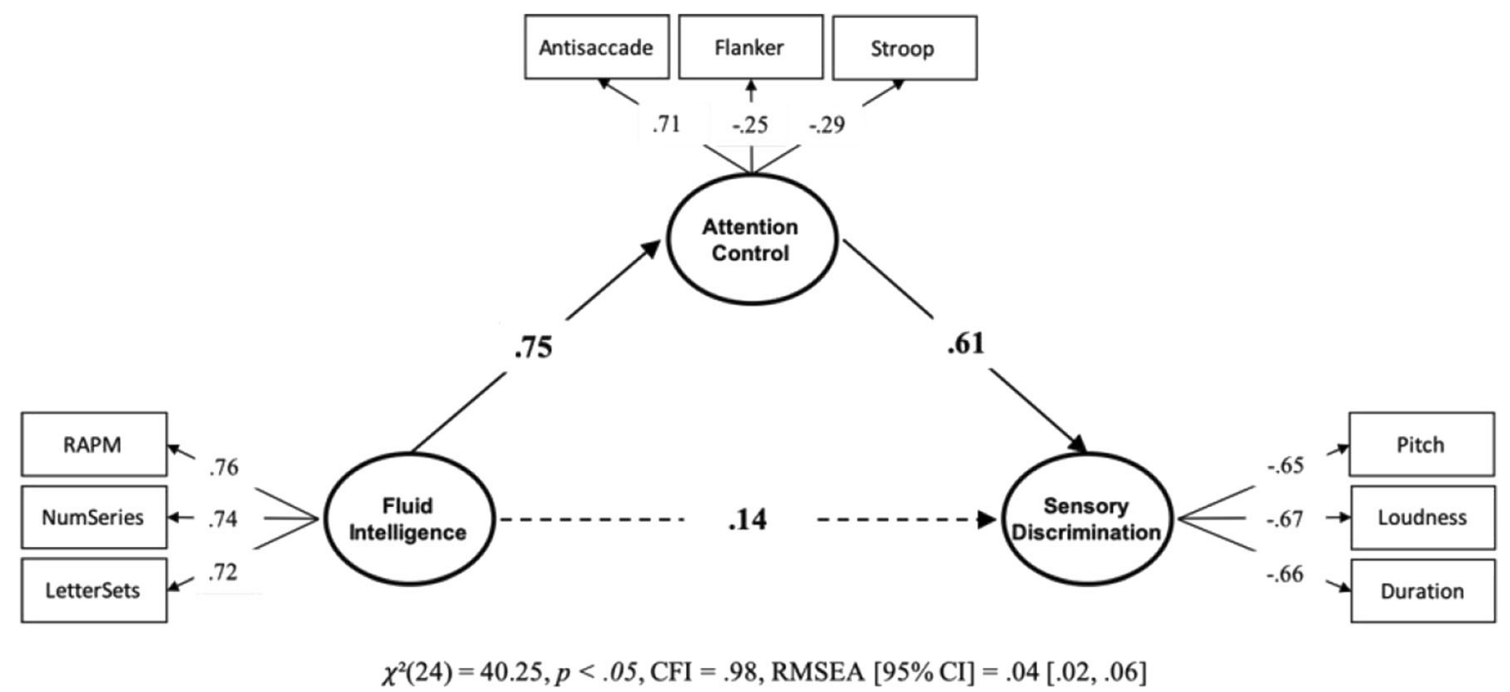

Fig. 13 Alternative structural equation models of Attention Control (with traditional Flanker and Stroop) mediating the Fluid Intelligence-Sensory Discrimination relationship in Study 2. N = 392

control with fluid intelligence and working-memory capacity. This provides discriminate validity for our interpretation that attention control drives performance across various sensory discrimination tasks and not vice versa.

While our results suggest an important role of attention control in sensory-discrimination ability and its relationship with fluid intelligence, they do not tell us anything about how attention control plays a role in sensory discrimination. The strong correspondence between attention control and sensory discrimination may seem surprising given that there are no distractors or prepotent response that are typical of situations that require the control of attention. In the Introduction, we defined attention control as the general ability to engage executive control functions that guide the focus of attention in a goal-directed manner. We also stated that individual differences in the ability to control attention manifest when automatic processes are in conflict with goal-oriented processes. Yet, such conflict appears to be absent in simple sensory discrimination tasks.

Identifying the stages at which attention is essential during performance of simple sensory discrimination tasks can help to elucidate the role of attention control. Due to the nature of how we administered the visual versus auditory discrimination tasks, the processes may differ slightly between the two sets of tasks. In the auditory discrimination tasks from Study 1 and 2, we presented the two to-be discriminated tones sequentially separated by $500 \mathrm{~ms}$, and each tone only occurred for a brief duration. First of all, encoding of the first tone will depend on the intensity and selectivity of attention. If there occurs a lapse of attention, such as in the form of mind-wandering, just before or during the first tone, then encoding the quality (pitch, loudness, or duration) of the first tone will lead

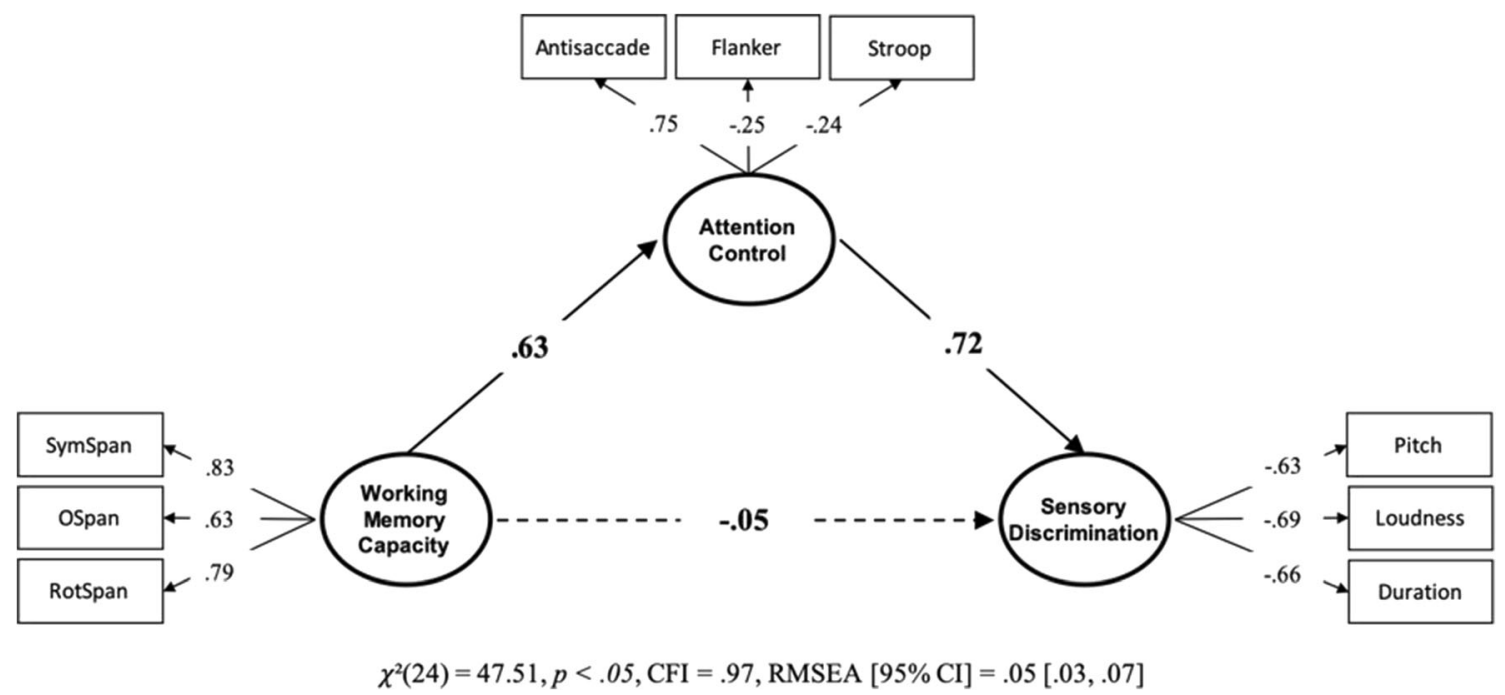

Fig. 14 Alternative structural equation models of Attention Control (with traditional Flanker and Stroop) mediating the Working-Memory Capacity Sensory Discrimination relationship in Study 2. N = 392 
Table 11 Test-retest correlations. $\mathrm{N}=63$

\begin{tabular}{ll} 
Task & Test-retest correlation \\
\hline Duration & 0.53 \\
Loud & 0.52 \\
Pitch & 0.81 \\
\hline
\end{tabular}

to a poor representation. Such lapses of attention occur due to executive control failures to maintain task goals, and the frequency of such lapses is correlated with individual differences in working-memory capacity and attention control (McVay \& Kane, 2009; Unsworth \& McMillan, 2014). After encoding of the first tone, the representation of the tone must be maintained in working memory to be compared with the second tone. If the first tone is not maintained, the representation may decay or face interference from internal distractions (mind-wandering) and external distractions (noises in the environment). Then, encoding the second tone will, again, depend on maintaining task goals and reducing interference from internal and external distractions. Finally coming to the actual act of discrimination, the difference in quality of the two tones needs to be compared in working memory. The ease and success of making this comparison will depend on all the prior steps just outlined. Essentially, the more focused or attentive one is on a trial-by-trial basis the finer sensory discriminations they will be able to make.

While this account suggests that internal distractors are a major source of individual differences in attention control on sensory discrimination performance, prior research on mindwandering suggest only a small to moderate relationship of mind-wandering with working-memory capacity and

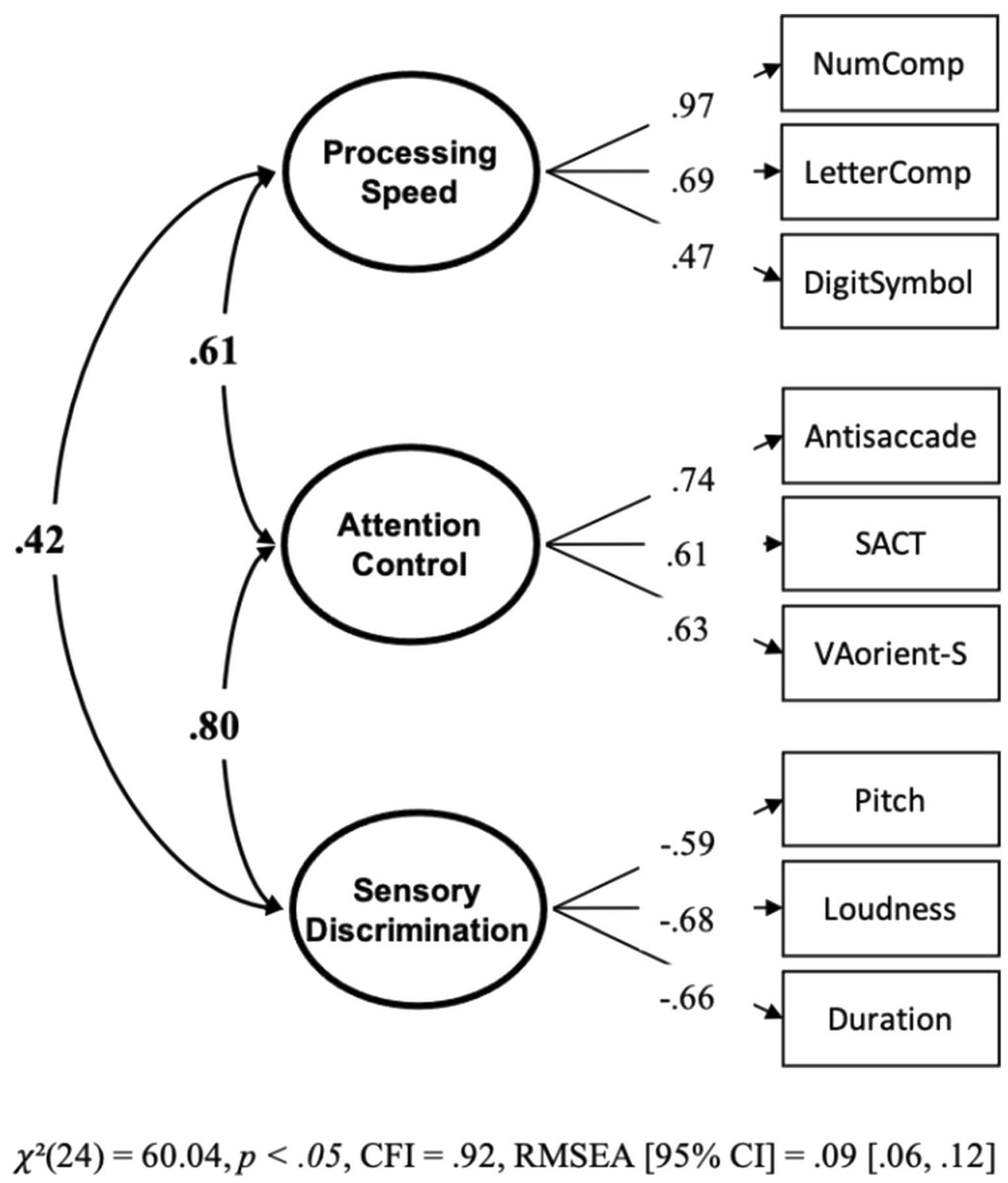

Fig. 15 Confirmatory factory analysis with processing speed, attention control, and sensory discrimination. $\mathrm{N}=176$ 


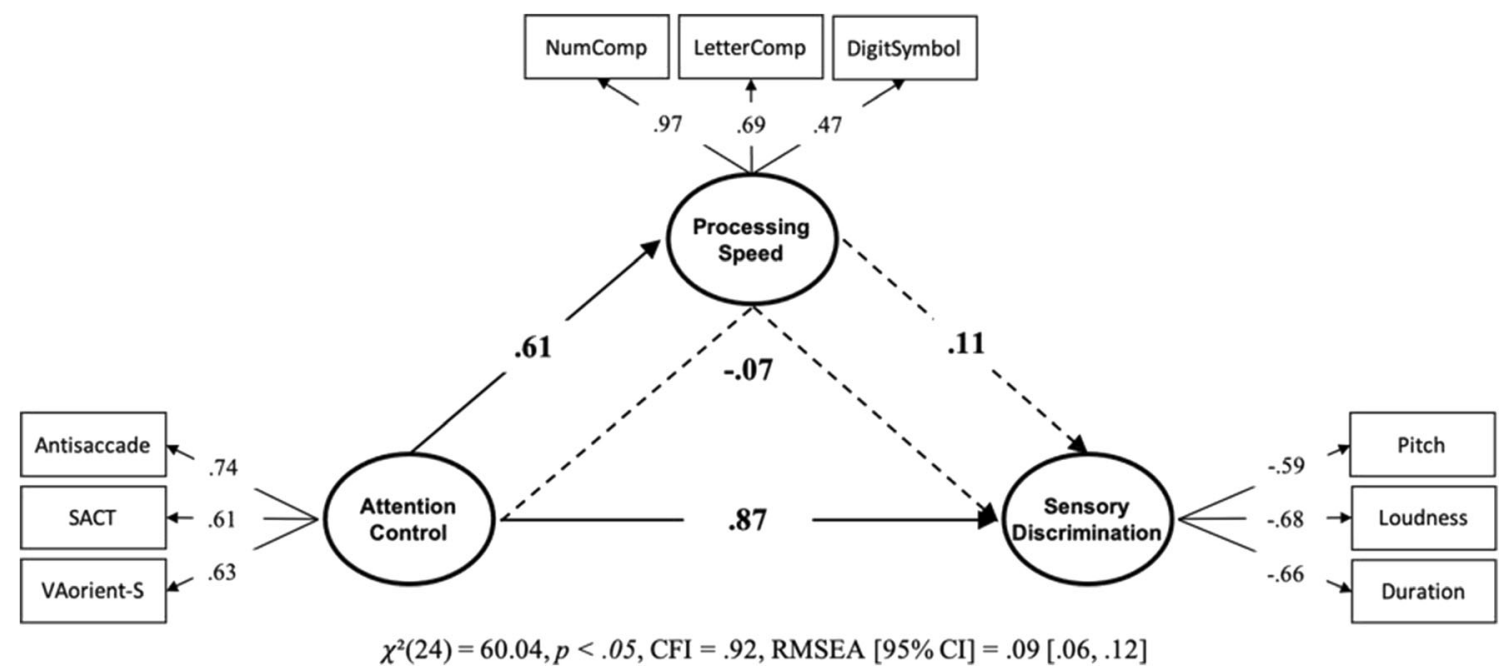

Fig. 16 Structural equation model with (a) attention control predicting sensory discrimination, and (b) processing speed included as a mediator. There are two paths from attention control to sensory discrimination. The direct path is the solid line with a value of .87 . The indirect path is the dotted line, with a value of -.07 , that goes through processing speed first then sensory discrimination. This represents the effect of attention control on sensory discrimination that can be explained by processing speed. All dotted lines are non-significant paths. $\mathrm{N}=176$ attention control (McVay \& Kane, 2009; Robison et al., 2017). Therefore, it would not be expected that internal distraction would fully account for the relationship between attention control and sensory discrimination.

Another possibility has to do with role of attention at the stage of encoding the stimuli. The role of attention on early and late perceptual stages of processing is well studied in the neurophysiological and neuroimaging literatures (Deco \& Rolls, 2005; Kok, 1997; Luck et al., 2000; O’Craven et al., 1997; Usher \& Niebur, 1996). One of the most influential theories, biased competition theory, states that attention to one stimulus or stimulus feature biases the neural activation in neurons that respond to that stimulus or stimulus feature (Desimone \& Duncan, 1995). This bias is not only observed by an increased activation in the relevant brain areas but an inhibition of activity in nearby non-relevant brain areas, thereby increasing the signal-to-noise ratio of attended stimuli over unattended stimuli. Most neural models of top-down attention on perception are based on similar principles as the biased competition theory (Usher \& Niebur, 1996). The basic idea is that attention to a stimulus or stimulus feature enhances its activation and thereby representation of the stimulus for later stages of processing (Kok, 1997; O'Craven et al., 1997; Treue \& Martinez-Trujillo, 2006). Accordingly, in performing a sensory discrimination task, greater attention to the tone stimuli (over other sounds and visual objects) will enhance the neural activation associated with the tones and thereby lead to greater discrimination between the two tones (for an example of how attention can enchance discrimination, see Pestilli \& Carrasco, 2005). Therefore, we are suggesting that even in the absence of task-relevant distractors or prepotent responses, attention control biases early sensory processing by increasing signalto-noise ratio and thereby resulting in greater sensory discrimination. Specifically, those with higher attention control are

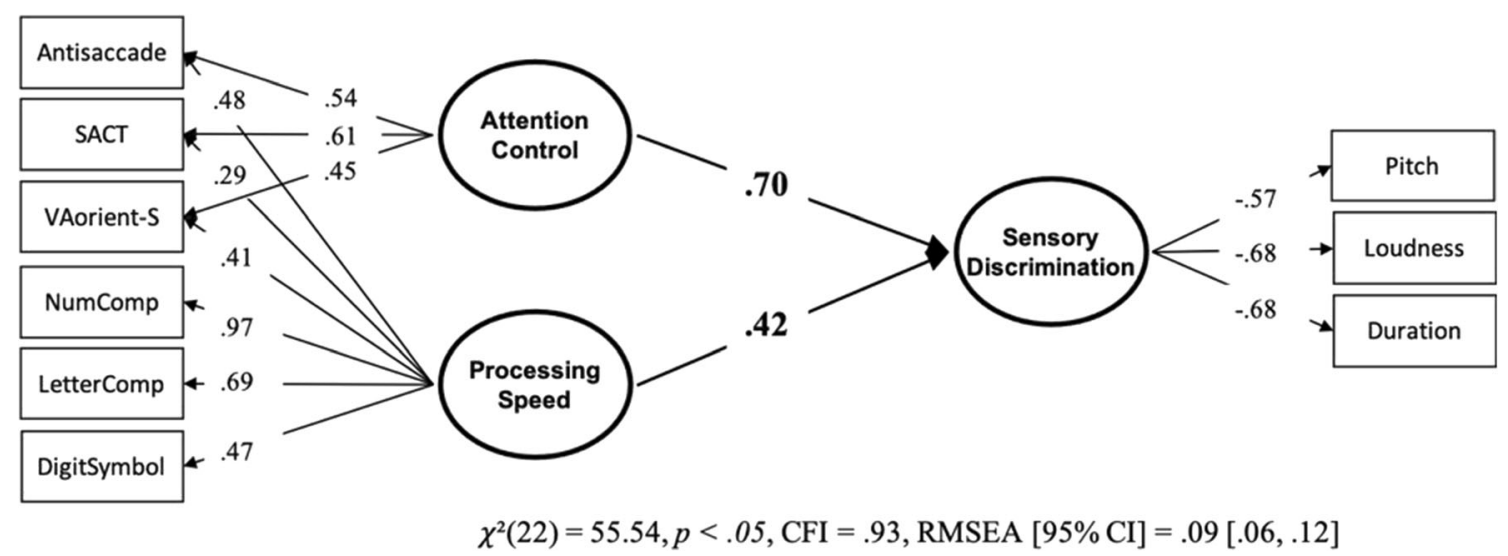

Fig. 17 Structural equation model extracting variance common to processing speed out of the attention control tasks. Attention control is still the stronger predictor of sensory discrimination. $\mathrm{N}=176$ 


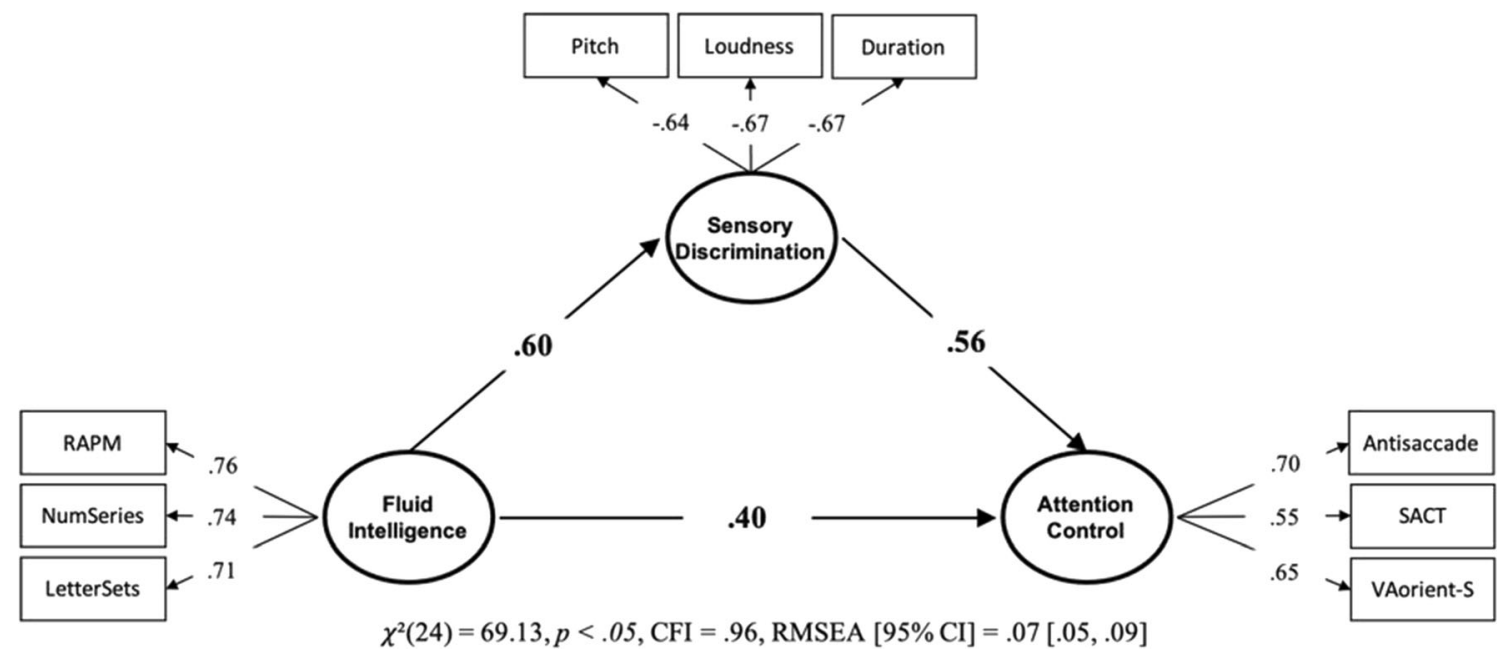

Fig. 18 Sensory discrimination does not fully mediate the relationship between fluid intelligence and attention control. $\mathrm{N}=392$

more focused, or more closely applying attention, to the target stimuli during encoding, leading to being able to make finer discriminations.

Whether attention-control processes are needed for a task depends largely on the demands a task places on the individual. For instance, if a sensory discrimination trial is easy for an individual (as might be the case for the first few trials in an adaptive task), then they may be able to rely on only perceptual attention without the need for controlled processing. However, if a sensory discrimination trial is difficult (as will be the case on the last trials in an adaptive task), then they may engage in controlled processing to enhance perceptual attention in order to make a finer discrimination between stimuli. If this is the case, this has implications as to the nature of adaptive tasks. It is possible that adaptive tasks reflect at least two components: (1) a threshold for the individual on the dimension the task is measuring (e.g., pitch discrimination), and (2) the individual's ability to engage in controlled processing. Our findings suggest that the threshold score estimated on each adaptive task contains dimension-specific (e.g., pitch vs. loudness) variance and common domain-general variance due to individual differences in attention control.

The strong role of attention control in sensory discrimination suggested by our findings raises the question of how attention control plays a role in other seemingly simple sensory tasks. When a revival of interest in the sensory discrimination-intelligence relationship occurred starting in the 1980 s, researchers were trying to understand the mechanisms underlying general intelligence (Acton \& Schroeder, 2001; Helmbold et al., 2006; Lynn et al., 1989; Raz et al., 1983, 1987, 1990; Watson, 1991). At around the same time, independent of intelligence research, a group of researchers were developing theoretical models of inspection time and tasks to measure it (Deary \& Stough, 1996). The inspection time tasks are essentially sensory discrimination tasks with a "speed of processing" component. In general, the inspection time tasks require a subject to discriminate the difference between two stimuli (two lines) presented simultaneously but

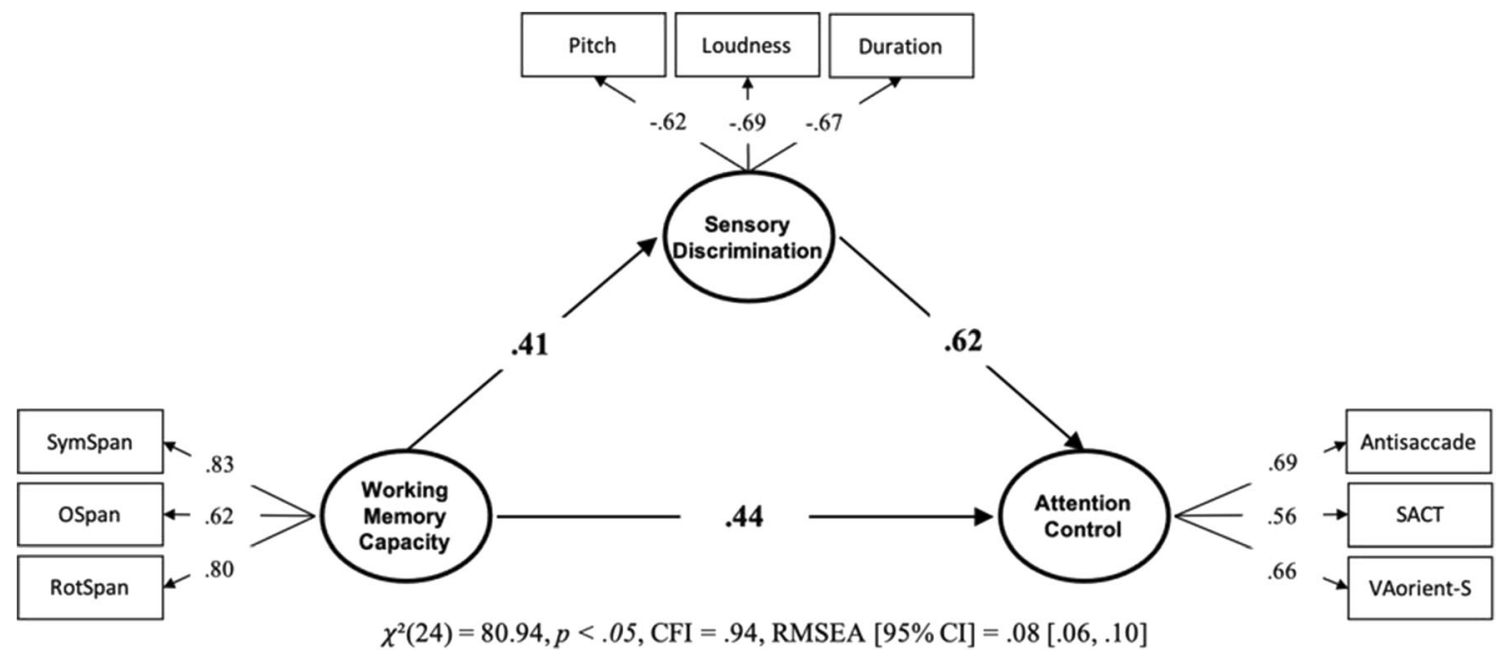

Fig. 19 Sensory discrimination does not fully mediate the relationship between working-memory capacity and attention control. $\mathrm{N}=392$ 
very briefly. The dependent measure for the task is usually the length of presentation at which the subject can perform at a given level of accuracy. Therefore, performance converges on a duration of presentation rather than a difficulty of discrimination. Researchers found that inspection time correlated with general intelligence (Deary \& Stough, 1996). Some researchers argued that the reason for this was the relation of sensory discrimination to both inspection time and intelligence (Irwin, 1984; Raz et al., 1987). Others argued that inspection time was primarily a measure of "mental speed" and was related to intelligence independently of sensory discrimination (Deary, 1994a; Deary \& Stough, 1996). This led researchers to propose "mental speed" - which had its basis in speed of how fast neurons can fire - as a fundamental cause in individual differences in intelligence (Deary \& Stough, 1996). It would be interesting to know the extent to which attention control plays a role in the inspection time-intelligence relationship as well. We believe the results would be similar to the current study. We suggest executive control functions are not only important in higher-order cognitive abilities, but also in lower-level cognitive and perceptual processes, such as "mental speed." However, this would require further investigation utilizing a variety of methodological and statistical techniques. Given our results, further investigation into the role of attention control in the inspection time-intelligence relationship is warranted.

If attention control plays such a prominent role in the sensory discrimination tasks used in this study, this has some important implications for the psychophysics of measuring sensory thresholds. What this suggests is that adaptive procedures to measure an individual's sensory threshold are not process pure. They do not purely measure a basic physiological characteristic of the individual's sensory systems, but rather they also capture considerable variance associated with top-down attention processes. Weighted up-down adaptive procedures assume that performance will eventually converge on some "true" threshold value (Kaernbach, 1991); however, performance on each trial will be influenced by top-down processes that maintain relevant information while inhibiting internal distractors ("mind wandering") and external distractors ("noise in the environment or task"). Performance on each trial, then, will be influenced by both state (arousal, focus, motivation) and trait (attention control, sense acuity) factors. We are not suggesting that using adaptive procedures to estimate an individual's sensory system is flawed or should be discarded. Quite the opposite - adaptive procedures avoid the problem of aggregating performance over many trials and thereby likely reduce (though not eliminate) noise from state level factors. Adaptive procedures can also avoid issues with the unreliability of reaction-time difference scores. Also, because adaptive sensory threshold procedures are reflective of top-down processes, this provides a potential method to investigate attention control. We simply point out that ignoring these higher-level processes in adaptive sensory threshold procedures can be problematic and theoretically misleading.

\section{Controlling for confounds}

Before moving on to the limitations of the present studies, we would like to discuss a more general concern. One of the most difficult challenges in psychology is determining whether we are measuring the psychological variables we say we are. In experimental psychology, this is referred to as internal validity; in differential psychology this is more commonly referred to as construct validity. Confounding variables are one threat to internal/construct validity. In experimental research, confounding variables are dealt with by carefully designing experimental manipulations that do not also manipulate the confounding variable. In individual differences research, there are many factors and traits related to the individual that we cannot manipulate and therefore it can be harder to control for confounding variables. The principal way of controlling for confounds, then, is to measure potentially confounding variables and statistically control for individual variation due to the confounding variable. If the variable of interest is still predictive, over and above confounding variables, then this provides what is referred to as incremental validity.

The reason for this discussion is that we have received a lot of reviewer criticism on the construct validity of our latent constructs. Specifically, the concerns are related to our attention control latent construct. One concern is that our attention control tasks are confounded with processing speed or just general task-ability-related variance. Another concern is that all the attention control tasks, to some extent, rely on processes of sensory discrimination. These are legitimate concerns about confound variables; however, to the best of our ability with the current data set, we have already addressed these concerns by partitioning shared and unique variance in different ways to provide incremental validity.

The model depicted in Fig. 17 is a good example of this. We label the predictive latent factors as "attention control" and "processing speed." However, in some ways this may be a misnomer. The "processing speed" factor is actually just a "general" factor that is explaining shared variance amongst both the processing speed and attention control tasks. Therefore, any shared variance due to processing speed, general task ability, motivation, or effort should be accounted for in this "general" factor. The "attention control" factor is actually "unique" variance of the attention tasks independent of variance shared with the processing speed tasks. In addition, the processing speed tasks are very similar to sensory discrimination tasks (see the Methods section). Yet, the "general" factor only predicted about $18 \%$ of variance in sensory discrimination and the "unique" factor predicted about $50 \%$ of variance in sensory discrimination. This model provides strong evidence that it is attention control related variance 
(and not processing speed or other sources of variance common across all tasks) that strongly predicts individual differences in sensory discrimination. The models depicted in Figs. 18 and 19 also provide evidence against the argument that sensory discrimination is the fundamental process underlying the relation between fluid intelligence, working-memory capacity, and attention control.

Finally, some reviewers have had issue with the inclusion of the selective visual arrays task as an attention-control measure because the conventional view is that this largely dominated by storage-capacity related variance. Besides the fact we replicated our finding in Study 2 without using the visual arrays task (see Figs. 13 and 14), in a separate article we provide strong evidence for an argument based on findings from the behavioral and neurophysiological literatures as well as on empirical findings in our lab from four different data sets collected over the last 10 years with $\mathrm{N}>1,500$ that the selective visual arrays task used in the current study is a better measure of attention control than memory capacity (Martin et al., 2019; available at https://psyarxiv.com/u92 cm/)

We now summarize our argument and findings that the selective visual arrays is a valid indictor of individual differences in attention control. First of all, it should be noted that the version of the visual arrays task used in the current study involves an attentional selection/filtering component. Subjects were presented with both red and blue rectangles but were told beforehand to only attend to red or blue (see the task description for more details).

This view is supported by studies from Vogel and colleagues that show that attention control processes to filter out irrelevant distractor items in the visual arrays can distinguish high- and low-capacity individuals (Drummond et al., 2012; Fukuda et al., 2015; Fukuda \& Vogel, 2009; Jost et al., 2011; Lee et al., 2010; Vogel et al., 2005). Contralateral delay activity (CDA; an EEG event-related potential) has been shown to be sensitive to the number of items currently held in working memory, as measured by a non-selective version of the visual arrays task (Vogel \& Machizawa, 2004). The non-selective versions of the task require attending to all items in the array, no selection/filtering of items is required. When a selection/filtering component was added to the visual arrays task (selectively attend to items on one side or, as in this study, items in one color), Vogel and colleagues showed that high capacity $(k)$ individuals showed the same CDA magnitude to arrays with two memory items only (e.g., two red rectangles) and two items with two distractors (e.g., two red rectangles and two blue rectangles; Vogel et al., 2005). This finding indicates that high-capacity individuals effectively filtered out the two distractor items and did not store them in working memory. Low capacity $(k)$ individuals, however, showed the same CDA magnitude to arrays with four memory items only (e.g., four red rectangles) and two items with two distractors (e.g., two red rectangles and two blue rectangles); therefore, low-capacity individuals did not effectively filter out the two distractor items, thereby storing them in working memory. This provides some of the most direct evidence for a mechanistic role of attention control in visual arrays capacity and in the same version of the task we used in the current study.

In Martin et al., (2019), we provide a set of analyses replicated over four large-scale studies totaling over 1,500 subjects that strongly support our argument. The visual arrays task strongly preferred to load onto an attention control factor rather than a working-memory capacity factor. Even when we cross-loaded the visual arrays task onto both an attention control and working-memory capacity factor, the loading to working-memory capacity was small and non-significant. In fact, the data from the current study were from one of those four large-scale studies. Therefore, we can conclude that in the current data set the visual arrays task reflects a domain-general ability to control attention and not a storage capacity limit in working memory for domain-specific visual events.

\section{Limitations}

We would now like to consider some limitations of the two studies. While the correlation between attention control and sensory discrimination in Study 1 was equivalent to $r=1$, in Study 2 the correlation was only $r=.79$. Besides differences in sample characteristics (as discussed in the Discussion section for Study 2), there were important differences in the sensory discrimination tasks used between the two studies. In the visual discrimination tasks (Study 1 only), the stimuli were presented simultaneously, and subjects had unlimited time to focus their attention on each stimulus and make a discrimination. In the auditory discrimination tasks, however, the stimuli were presented sequentially for only $500 \mathrm{~ms}$ with a $500-\mathrm{ms}$ interval between tones. Therefore, there was limited time to encode and compare the stimuli. The auditory discrimination tasks, then, have a greater demand on maintaining a representation of the first stimulus during the 500-ms interval; in addition, there was a greater demand on their focus of attention while the stimulus was occurring. In the visual discrimination tasks, there still may be a demand on maintaining the representation of one stimulus as attention shifts focus to the other stimulus (though still less compared to the auditory tasks). This lesser demand on executive control processes in the visual discrimination tasks may be why they had smaller loadings on the sensory-discrimination factor compared to the auditory discrimination tasks (Fig. 4); there was less common variance attributable to attention control in the visual discrimination tasks than the auditory discrimination tasks.

Given that in Study 2 the correlation between attention control and auditory discrimination was less than $r=1$, this suggests that there may indeed be variability in domainspecific sensory discrimination that is not accounted for by attention control. However, this domain-specific variance, as 
evidenced by the mediation models, is not correlated with intelligence or working-memory capacity. To more precisely determine the causal mechanisms of attention control versus domain-specific sensory abilities in sensory discrimination tasks may require combining differential and experimental methods; an interesting but resource-demanding prospect for future research.

\section{Conclusion}

The strong relationship between sensory discrimination and intelligence is what initially led Charles Spearman to formulate his theory on general intelligence that would go on to influence the field for decades to come. Spearman hypothesized that a central "Function" underlying differences in intelligence also plays a prominent role in sensory discrimination; "I take both sensory discrimination and the manifestations leading a teacher to impute general intelligence to be based on some deeper fundamental cause" (Deary, 1994b, p. 105).

What we have shown in two studies is that attention control plays a prominent role in sensory discrimination and is able to fully account for the relationship between sensory discrimination and intelligence. That is, the reason why a basic sensory task like sensory discrimination correlates with a high-order factor of general intelligence is due to individual differences in ability to control the selectivity and intensity of attention.

It is time we start paying more attention to this seminal and theoretically important relationship. It should be noted that these two studies are only correlational in nature; and if we are to understand the mechanisms underlying intelligence, further research that combines the experimental and differential approaches will be needed.

Author note This work was supported by Office of Naval Research Grant N00014-09-1-0129 to Randall W. Engle. Data, analysis scripts, and results output are available at: https://osf.io/hsqru/

None of the experiments were preregistered.

\section{References}

Acton, G. S., \& Schroeder, D. H. (2001). Sensory discrimination as related to general intelligence. Intelligence, 29(29), 263-271. https:// doi.org/10.1016/S0160-2896(01)00066-6

Aston-Jones, G., \& Cohen, J. D. (2005). An integrative theory of locus coeruleus-norepinephrine function: Adaptive gain and optimal performance. Annual Review of Neuroscience, 28, 403-450. https://doi. org/10.1146/annurev.neuro.28.061604.135709

Baron, R. M., \& Kenny, D. A. (1986). The moderator-mediator variable distinction in social psychological research: conceptual, strategic, and statistical considerations. Journal of Personality and Social Psychology https://doi.org/10.1037/0022-3514.51.6.1173
Barrett, L. F., Tugade, M. M., \& Engle, R. W. (2004). Individual differences in working-memory capacity and dual-process theories of the mind. Psychological Bulletin, 130(4), 553-573. https://doi.org/10. 1037/0033-2909.130.4.553

Brown, R. E. (2016). Hebb and Cattell: The Genesis of the Theory of Fluid and Crystallized Intelligence. Frontiers in Human Neuroscience, 10(December). https://doi.org/10.3389/fnhum.2016. 00606

Browne, M. W., \& Cudeck, R. (1993). Alternative ways of assessing model fit. Sage Focus Editions, 154, 136.

Bryne, B. M. (1994). Structural equation modeling with EQS and EQS/ Windows. Thousand Oaks, Califomia: Sage Publications.

Conway, A. R. ., Kane, M. J., \& Engle, R. W. (1999). Is Spearman's g determined by speed or working-memory capacity? Psycoloquy, 10(074). https://doi.org/10.1088/1742-6596/1071/1/012003

Conway, A. R. A., Kane, M. J., Bunting, M. F., Hambrick, Z. D., Wilhelm, O., \& Engle, R. W. (2005). Working memory span tasks: A methodological review and user's guide. Psychonomic Bulletin \& Review, 12(5), 769-786.

Conway, A. R. A., Kane, M. J., \& Engle, R. W. (2003). Working memory capacity and its relation to general intelligence. Trends in Cognitive Sciences, 7(12), 547-552. https://doi.org/10.1016/j.tics.2003.10. 005

Cowan, N., Elliott, E. M., Saults, S. J., Morey, C. C., Mattox, S., Hismjatullina, A., \& Conway, A. R. A. (2005). On the capacity of attention: Its estimation and its role in working memory and cognitive aptitudes. Cognitive Psychology, 51, 42-100. https://doi.org/10. 1016/j.cogpsych.2004.12.001

Deary, I. J. (1994a). Intelligence and auditory discrimination: Seperating processing speed and fidelity of stimulus representation. Intelligence, 18, 189-213.

Deary, I. J. (1994b). Sensory discrimination and intelligence: Postmortem or resurrection? The American Journal of Psychology, 107(1), 95115.

Deary, I. J., Bell, J. P., Bell, A. J., Campbell, M. L., \& Fazal, N. D. (2004). Sensory Discrimination and Intelligence: Testing Spearman's Other Hypothesis. The American Journal of Psychology, 117(1), 1-18.

Deary, I. J., \& Stough, C. (1996). Intelligence and Inspection Time. American Psychologist, 5, 599-608.

Deco, G., \& Rolls, E. T. (2005). Neurodynamics of biased competition and cooperation for attention: A model with spiking neurons. Journal of Neurophysiology, 94(1), 295-313. https://doi.org/10. 1152/jn.01095.2004

Desimone, R., \& Duncan, J. (1995). Neural Mechanisms of Selective Visual Attention. Annual Review of Neuroscience, 18, 193-222. https://doi.org/10.1146/annurev.neuro.18.1.193

Dinges, D. F., \& Powell, J. W. (1985). Microcomputer analyses of performance on a portable, simple visual RT task during sustained operations. Behavior Research Methods, Instruments, \& Computers, 17(6), 652-655. https://doi.org/10.3758/BF03200977

Draheim, C., Harrison, T. L., Embretson, S. E., \& Engle, R. W. (2018). What item response theory can tell us about the complex span tasks. Psychological Assessment, 30(1), 116-129. https://doi.org/10.1037/ pas0000444

Draheim, C., Hicks, K. L., \& Engle, R. W. (2016). Combining reaction time and accuracy: The relationship between working-memory capacity and task switching as a case example. Perspectives on Psychological Science, 11(1), 133-155. https://doi.org/10.1177/ 1745691615596990

Draheim, C., Mashburn, C. A., Martin, J. D., \& Engle, R. W. (2019). Reaction time in differential and developmental research: A review and commentary on the problems and alternatives. Psychological Bulletin, 145, 508-535.

Draheim, C., Tsukahara, J. S., Martin, J. D., Mashburn, C. A., \& Engle, R. W. (2020). A toolbox approach to improving the measurement of 
attention control. Preprint on PsyArXiv. https://psyarxiv.com/ q985d/

Drummond, S. P. A., Anderson, D. E., Straus, L. D., Vogel, E. K., \& Perez, V. B. (2012). The effects of two types of sleep deprivation on visual working-memory capacity and filtering efficiency. PLoS ONE, 7(4), 1-8. https://doi.org/10.1371/journal.pone.0035653

Egeth, H. E., \& Yantis, S. (1997). Visual attention: control, representation, and time course. Annual Review of Psychology, 48, 269-297. https://doi.org/10.1146/annurev.psych.48.1.269

Ekstrom, R., French, J., Harman, H., \& Dermen, D. (1976). Manual for kit of factor-referenced cognitive tests. In Princeton NJ Educational Testing Service (Vol. 102). https://doi.org/10.1073/pnas. 0506897102

Engle, R. W. (2002). Working memory capacity as executive attention. Current Directions in Psychological Science, 11(1), 19-23. https:// doi.org/10.1111/1467-8721.00160

Engle, R. W. (2018). Working Memory and Executive Attention: A Revisit. Perspectives on Psychological Science, 13(2), 190-193. https://doi.org/10.1177/1745691617720478

Engle, R. W., \& Martin, J. D. (2018). Is a Science of the Mind Even Possible? Reply to Logie (2018). Journal of Applied Research in Memory and Cognition, 7(4), 493-498. https://doi.org/10.1016/j. jarmac.2018.10.002

Friedman, N. P., \& Miyake, A. (2004). The relations among inhibition and interference control functions: A latent-variable analysis. Journal of Experimental Psychology: General, 133(1), 101-135. https://doi.org/10.1037/0096-3445.133.1.101

Fukuda, K., \& Vogel, E. K. (2009). Human variation in overriding attentional capture. The Journal of Neuroscience, 29(27), 8726-8733. https://doi.org/10.1523/JNEUROSCI.2145-09.2009

Fukuda, K., Woodman, G. F., \& Vogel, E. K. (2015). Individual Differences in Visual Working Memory Capacity. Mechanisms of Sensory Working Memory, 105-119. https://doi.org/10.1016/b9780-12-801371-7.00009-0

Galton, F. (1883). Inquiries into human faculty and its development. In Inquiries into human faculty and its development. JM Dent and Company.

Hairston, W. D., \& Maldjian, J. a. (2009). An adaptive staircase procedure for the E-Prime programming environment. Computer Methods and Programs in Biomedicine, 93(1), 104-108. https:// doi.org/10.1016/j.cmpb.2008.08.003

Hallett, P. E. (1978). Primary and secondary saccades to goals defined by instructions. Vision Research, 18, 1279-1296.

Hebb, D. O. (1942). The effect of early and late brain injury upon test scores, and the nature of normal adult intelligence. Proceedings of the American Philosophical Society, 85(3), 275-292.

Hedge, C., Powell, G., \& Sumner, P. (2018). The reliability paradox: Why robust cognitive tasks do not produce reliable individual differences. Behavior Research Methods, 50(3), 1166-1186. https:// doi.org/10.3758/s13428-017-0935-1

Heitz, R. P., Redick, T. S., Hambrick, D. Z., Kane, M. J., Conway, A. R. A., \& Engle, R. W. (2006). Working memory, executive function, and general fluid intelligence are not the same. Behavioral and Brain Sciences, 29(02), 135-136.

Heitz, R. P., Unsworth, N., \& Engle, R. W. (2005). Working memory capacity, attention control, and fluid intelligence. In Handbook of Understanding and Measuring Intelligence (pp. 61-77). https://doi. org/10.1109/ICCASM.2010.5622177

Helmbold, N., Troche, S., \& Rammsayer, T. (2006). Temporal information processing and pitch discrimination as predictors of general intelligence. Canadian Journal of Experimental Psychology, 60(4), 294-306. https://doi.org/10.1037/cjep2006027

Hu, L. T., \& Bentler, P. M. (1999). Cutoff criteria for fit indexes in covariance structure analysis: Conventional criteria versus new alternatives. Structural Equation Modeling, 6(1), 1-55. https://doi. org/10.1080/10705519909540118
Hughes, M. M., Linck, J. A., Bowles, A. R., Koeth, J. T., \& Bunting, M. F. (2014). Alternatives to switch-cost scoring in the task-switching paradigm: Their reliability and increased validity. Behavior Research Methods, 46(3), 702-721. https://doi.org/10.3758/ s13428-013-0411-5

Hutchison, K. A. (2007). Attentional control and the relatedness proportion effect in semantic priming. Journal of Experimental Psychology: Learning Memory and Cognition, 33(4), 645-662. https://doi.org/10.1037/0278-7393.33.4.645

Irwin, R. J. (1984). Inspection time and its relation to intelligence. Intelligence, 8(1), 47-65.

James, W. (1890). The principles of psychology (Vol. 1, Issue 2). Harvard University Press.

Jost, K., Bryck, R. L., Vogel, E. K., \& Mayr, U. (2011). Are old adults just like low working memory young adults? Filtering efficiency and age differences in visual working memory. Cerebral Cortex, 21, 1147-1154. https://doi.org/10.1093/cercor/bhq185

Kaernbach, C. (1991). Simple adaptive testing with the weighted updown method. Perception \& Psychophysics, 49(3), 227-229.

Kahneman, D. (1973). Attention and effort. Prentice-Hall. https://doi.org/ $10.2307 / 1421603$

Kane, H., \& Brand, C. (2003). The Importance of Spearman's g as a Psychometric, Social and Educational Construct. The Occidental Quaterly, 3(1), 7-30. https://courseresources.derby.ac.uk/ bbcswebdav/pid-1404135-dt-content-rid-11501238 1/courses/ 2014-SPR-5ED506/spearmananded.pdf

Kane, M. J., Conway, A. R. A., Bleckley, M. K., \& Engle, R. W. (2001). A controlled-attention view of working-memory capacity. Journal of Experimental Psychology: General, 130(2), 169-183. https://doi. org/10.1037//0096-3445.130.2.169

Kane, M. J., \& Engle, R. W. (2003). Working-memory capacity and the control of attention: the contributions of goal neglect, response competition, and task set to Stroop interference. Journal of Experimental Psychology: General, 132(1), 47-70. https://doi.org/10.1037/00963445.132.1.47

Kane, M. J., Hambrick, D. Z., \& Conway, A. R. A. (2005). Working memory capacity and fluid intelligence are strongly related constructs: comment on Ackerman, Beier, and Boyle (2005). Psychological Bulletin, 131(1), 66-71. https://doi.org/10.1037/ 0033-2909.131.1.66

Kenny, D. A. (2015). Measuring Model Fit. http://davidakenny.net/cm/ fit.htm

Kok, A. (1997). Event-related-potential (ERP) reflections of mental resources: A review and synthesis. Biological Psychology, 45(1-3), 19-56. https://doi.org/10.1016/S0301-0511(96)05221-0

Kovacs, K., \& Conway, A. R. . (2016). Process Overlap Theory: A Unified Account of the General Factor of Intelligence. Psychological Inquiry, 27(3), 151-177. https://doi.org/10.1080/ 1047840X.2016.1153946

Kyllonen, P. C., \& Christal, R. E. (1990). Reasoning ability is (little more than) working-memory capacity?! Intelligence, 14(4), 389-433.

Lee, E. Y., Cowan, N., Vogel, E. K., Rolan, T., Valle-Inclán, F., \& Hackley, S. A. (2010). Visual working memory deficits in patients with Parkinson's disease are due to both reduced storage capacity and impaired ability to filter out irrelevant information. Brain, 133, 2677-2689. https://doi.org/10.1093/brain/awq197

Luck, S. J., \& Vogel, E. K. (1997). The capacity of visual working memory for features and conjunctions. Nature, 390, 279-284. https://doi.org/10.1038/36846

Luck, S. J., Woodman, G. F., \& Vogel, E. K. (2000). Event-related potential studies of attention. Trends in Cognitive Sciences, 4(11), 432-440. https://doi.org/10.1016/S1364-6613(00)01545-X

Lynn, R., Wilson, R. G., \& Gault, A. (1989). Simple musical tests as measures of Spearman's g. Personality and Individual Differences.

Martin, J. D., Tsukahara, J. S., Mashburn, C. A., Draheim, C., Shipstead, Z., Vogel, E. K., \& Engle, R. W. (2019). The visual arrays task: 
Visual storage capacity or attention control? Preprint on PsyArXiv. https://psyarxiv.com/u92cm/

McVay, J. C., \& Kane, M. J. (2009). Conducting the train of thought: Working memory capacity, goal neglect, and mind wandering in an executive-control task. Journal of Experimental Psychology: Learning, Memory, and Cognition, 35(1), 196-204. https://doi. org/10.1037/a0014104

Meyer, C. S., Hagmann-von Arx, P., Lemola, S., \& Grob, A. (2010). Correspondence Between the General Ability to Discriminate Sensory Stimuli and General Intelligence. Journal of Individual Differences, 31, 46-56. https://doi.org/10.1027/1614-0001/a000006

Miller, A. L., Gross, M. P., \& Unsworth, N. (2019). Individual differences in working-memory capacity and long-term memory: The influence of intensity of attention to items at encoding as measured by pupil dilation. Journal of Memory and Language, 104(January 2018), 25-42. https://doi.org/10.1016/j.jml.2018.09.005

Miller, J., \& Ulrich, R. (2013). Mental chronometry and individual differences: Modeling reliabilities and correlations of reaction time means and effect sizes. Psychonomic Bulletin and Review, 20(5), 819-858. https://doi.org/10.3758/s13423-013-0404-5

Miyake, A., Friedman, N. P., Emerson, M. J., Witzki, A. H., Howerter, A., \& Wager, T. D. (2000). The unity and diversity of executive functions and their contributions to complex "Frontal Lobe" tasks: a latent variable analysis. Cognitive Psychology, 41(1), 49-100. https://doi.org/10.1006/cogp.1999.0734

Norman, D. A., \& Shallice, T. (1986). Attention to action. Willed and automatic control of behavior. In Consciousness and SelfRegulation (pp. 1-18). Springer.

O’Craven, K. M., Rosen, B. R., Kwong, K. K., Treisman, A., \& Savoy, R. L. (1997). Voluntary attention modulates fMRI activity in human MT-MST. Neuron, 18(4), 591-598. https://doi.org/10.1016/S08966273(00)80300-1

Oberauer, K., Süß, H. M., Wilhelm, O., \& Wittman, W. W. (2003). The multiple faces of working memory: Storage, processing, supervision, and coordination. Intelligence, 31(2), 167-193. https://doi. org/10.1016/S0160-2896(02)00115-0

Paap, K. R., \& Oliver, S. (2016). The role of test-retest reliability in measuring individual and group differences in executive functioning. Journal of Neuroscience Methods, 274, 81-93. https://doi.org/ 10.1016/j.jneumeth.2016.10.002

Pestilli, F., \& Carrasco, M. (2005). Attention enhances contrast sensitivity at cued and impairs it at uncued locations. Vision Research, 45(14), 1867-1875. https://doi.org/10.1016/j.visres.2005.01.019

Posner, M. I., \& DiGirolamo, G. J. (1998). Conflict, target detection and cognitive control. in r. parasuraman (ed.), The attentive brain (pp. 401-423). Cambridge, MA: MIT Press.

Psychology Software Tools, Inc. [E-Prime 2.0]. (2016). https://www. pstnet.com

R Core Team. (2018). $R$ : A language and environment for statistical computing. R Foundation for Statistical Computing. https://www. r-project.org/

Rammsayer, T. (1992). Effects of benzodiazepine-induced sedation on temporal processing. Human Psychopharmacology: Clinical and Experimental, 7(5), 311-318. https://doi.org/10.1002/hup. 470070503

Raven, J., Raven, J. C., \& Court, J. (1998). Manual for Raven's progressive matrices and vocabulary scales. In Raven manual.

Raz, N., Moberg, P. J., \& Millman, D. (1990). Effects of age and agerelated differences in auditory information processing on fluid and crystallized intelligence. Personality and Individual Differences, 11(11), 1147-1152. https://doi.org/10.1016/0191-8869(90)90027$\mathrm{O}$

Raz, N., Willerman, L., Ingmundson, P., \& Hanlon, M. (1983). Aptituderelated differences in auditory recognition masking. Intelligence, 7 , 71-90. https://doi.org/10.1016/0160-2896(83)90021-1
Raz, N., Willerman, L., \& Yama, M. (1987). On sense and senses: Intelligence and auditory information processing. Personality and Individual Differences, 8(2), 201-210.

Redick, T. S., Unsworth, N., Kelly, A. J., \& Engle, R. W. (2012). Faster, smarter? Working memory capacity and perceptual speed in relation to fluid intelligence. Journal of Cognitive Psychology. https://doi. org/10.1080/20445911.2012.704359

Rey-Mermet, A., Gade, M., \& Oberauer, K. (2018). Should we stop thinking about inhibition? Searching for individual and age differences in inhibition ability. Journal of Experimental Psychology: Learning Memory and Cognition, 44(4), 501-526. https://doi.org/ $10.1037 / \mathrm{xlm} 0000450$

Rey-Mermet, A., Gade, M., Souza, A. S., von Bastian, C. C., \& Oberauer, K. (2019). Is Executive Control Related to Working Memory Capacity and Fluid Intelligence? Journal of Experimental Psychology: General. https://doi.org/10.1037/xge0000593

Reynolds, J. H., \& Pasternak, T. (2000). Attention Increases Sensitivity of V4 Neurons increases the magnitude of the neuronal response elic. Neuron, 26, 703-714. https://doi.org/10.1016/S0896-6273(00) 81206-4

Robison, M. K., Gath, K. I., \& Unsworth, N. (2017). The neurotic wandering mind: An individual differences investigation of neuroticism, mind-wandering, and executive control. The Quarterly Journal of Experimental Psychology, 70(4), 649-663. https://doi.org/10.1080/ 17470218.2016.1145706

Rosseel, Y. (2012). lavaan: An R package for structural equation modeling. Journal of Statistical Software, 48(2), 1-36.

Rueda, M. R. (2018). Attention in the heart of intelligence. Trends in Neuroscience and Education, 13(November), 26-33. https://doi. org/10.1016/j.tine.2018.11.003

Salthouse, T. A., \& Babcock, R. L. (1991). Decomposing Adult Age Differences in Working Memory. Developmental Psychology, 27(5), 763-776. https://doi.org/10.1037/0012-1649.27.5.763

Schoenfeld, M. A., Hopf, J. M., Martinez, A., Mai, H. M., Sattler, C., Gasde, A., Heinze, H. J., \& Hillyard, S. A. (2007). Spatio-temporal analysis of feature-based attention. Cerebral Cortex, 17(10), 24682477. https://doi.org/10.1093/cercor/bhl154

Schönbrodt, F. D., \& Perugini, M. (2013). At what sample size do correlations stabilize? Journal of Research in Personality, 47(5), 609 612. https://doi.org/10.1016/j.jrp.2013.05.009

Shipstead, Z., Harrison, T. L., \& Engle, R. W. (2016). Working memory capacity and fluid intelligence: Maintenance and disengagement. Perspectives on Psychological Science, 11(6), 771-799. https:// doi.org/10.1177/1745691616650647

Shipstead, Z., Lindsey, D. R. B., Marshall, R. L., \& Engle, R. W. (2014). The mechanisms of working-memory capacity: Primary memory, secondary memory, and attention control. Journal of Memory and Language, 72, 116-141. https://doi.org/10.1016/j.jml.2014.01.004

Spearman, C. (1904). "General intelligence," Objectively determined and measured. The American Journal of Psychology, 15(2), 201-292.

Stevens, S. S. (1975). Psychophysics: Introduction to its perceptual neural and social prospects. John Wiley.

Thurstone, L. L. (1938). Primary mental abilities.

Treue, S., \& Martinez-Trujillo, J. C. (2006). Visual search and single-cell electrophysiology of attention: Area MT, from sensation to perception. Visual Cognition, 14(4-8), 898-910. https://doi.org/10.1080/ 13506280500197256

Troche, S. J., Wagner, F. L., Voelke, A. E., Roebers, C. M., \& Rammsayer, T. H. (2014). Individual differences in workingmemory capacity explain the relationship between general discrimination ability and psychometric intelligence. Intelligence, 44, 4050. https://doi.org/10.1016/j.intell.2014.02.009

Unsworth, N., Heitz, R. P., Schrock, J. C., \& Engle, R. W. (2005). An automated version of the operation span task. Behavior Research Methods, 37(3), 498-505. https://doi.org/10.3758/BF03192720 
Unsworth, N., \& McMillan, B. D. (2014). Similarities and differences between mind-wandering and external distraction: A latent variable analysis of lapses of attention and their relation to cognitive abilities. Acta Psychologica, 150, 14-25. https://doi.org/10.1016/j.actpsy. 2014.04.001

Unsworth, N., \& Robison, M. K. (2017). A locus coeruleusnorepinephrine account of individual differences in workingmemory capacity and attention control. Psychonomic Bulletin \& Review, 1957. https://doi.org/10.3758/s13423-016-1220-5

Unsworth, N., \& Robison, M. K. (2020). Working memory capacity and sustained attention: A cognitive-energetic perspective. Journal of Experimental Psychology: Learning Memory and Cognition, 46(1), 77-103. https://doi.org/10.1037/xlm0000712

Usher, M., \& Niebur, E. (1996). Modeling the temporal dynamics of IT neurons in visual search: A mechanism for top-down selective attention. Journal of Cognitive Neuroscience, 8(4), 311-327. https:// doi.org/10.1162/jocn.1996.8.4.311
Vandierendonck, A. (2018). Further Tests of the Utility of Integrated Speed-Accuracy Measures in Task Switching. Journal of Cognition, 1(1), 1-16. https://doi.org/10.5334/joc.6

Vogel, E. K., \& Machizawa, M. G. (2004). Neural activity predicts individual differences in visual working-memory capacity. Nature, 428 , 748-751. https://doi.org/10.1038/nature02447

Vogel, E. K., McCollough, A. W., \& Machizawa, M. G. (2005). Neural measures reveal individual differences in controlling access to visual working memory. Nature, 438, 500-503.

Watson, B. U. (1991). Some relationships between intelligence and auditory discrimination. Journal of Speech, Language, and Hearing Research, 34(3), 621-627.

Wechsler, D. (1997). Wechsler Adult Intelligence Scale (3rd ed.). Psychological Corporation.

Publisher's note Springer Nature remains neutral with regard to jurisdictional claims in published maps and institutional affiliations. 